\title{
Statistical Study of Medium-Scale Traveling Ionospheric Disturbances in Low-Latitude Ionosphere Using an Automatic Algorithm
}

\section{PinHsuan Cheng}

National Cheng Kung University https://orcid.org/0000-0002-2658-4898

Charles Lin ( $\nabla$ charles@mail.ncku.edu.tw)

National Cheng Kung University No.1 https://orcid.org/0000-0001-8955-8753

\section{Yuichi Otsuka}

Nagoya University

Hanli Liu

High altitude observatory

\section{Panthalingal Krishanunni Rajesh}

National Cheng Kung University

\section{Chia-Hung Chen}

National Cheng Kung University

Jia-Ting Lin

National Cheng Kung University

\section{Full paper}

Keywords: low-latitude MSTIDs, Support Vector Machine, Atmospheric Gravity Waves, Sporadic E layers

Posted Date: December 30th, 2020

DOl: https://doi.org/10.21203/rs.3.rs-136037/v1

License: (9) This work is licensed under a Creative Commons Attribution 4.0 International License.

Read Full License 
1 Title: Statistical Study of Medium-Scale Traveling Ionospheric Disturbances in

2 Low-Latitude Ionosphere Using an Automatic Algorithm

3 Author \#1: Pin-Hsuan Cheng, National Cheng Kung University No.1, University

4 Road, Tainan City 701, Taiwan (R.O.C), p851102peter5@gmail.com

5 Author \#2: Charles Chien-Hung Lin, National Cheng Kung University No.1,

$6 \quad$ University Road, Tainan City 701, Taiwan (R.O.C), charles@mail.ncku.edu.tw

7 Author \#3: Yuichi Otsuka, Institute for Space-Earth Environmental Research, Nagoya

8 University Furo-cho, Chikusa-ku, Nagoya 464-8601, Japan, otsuka@isee.nagoya-

$9 \quad$ u.ac.jp

Author \#4: Hanli Liu, 1850 Table Mesa Dr, Boulder, CO USA 80305, liuh@ucar.edu

Author \#5 Panthalingal Krishanunni Rajesh, National Cheng Kung University No.1,

University Road, Tainan City 701, Taiwan (R.O.C), pkrgere@gmail.com

Author \#6: Chia-Hung Chen, National Cheng Kung University No.1, University

Road, Tainan City 701, Taiwan (R.O.C), koichi@mail.ncku.edu.tw

Author \#7: Jia-Ting Lin, National Cheng Kung University No.1, University Road,

Tainan City 701, Taiwan (R.O.C), familyiscute@gmail.com

\section{Indicate the corresponding author:}

Charles Lin 


\section{Abstract}

21 This study investigates the medium-scale traveling ionospheric disturbances (MSTIDs)

22 statistically at the low-latitude equatorial ionization anomaly (EIA) region in the

23 northern hemisphere. We apply the automatic detection algorithm including the three-

24 dimensional fast Fourier transform (3-D FFT) and support vector machine (SVM) on

25 total electron content (TEC) observations, derived from a network of ground-based

26 global navigation satellite system (GNSS) receivers in Taiwan $\left(14.5^{\circ} \mathrm{N}\right.$ geomagnetic

27 latitude; $32.5^{\circ}$ inclination), to identify MSTID from other waves or irregularity features.

28 The obtained results are analyzed statistically to examine the behavior of low-latitude

29 MSTIDs. Statistical results indicate the following characteristics. First, the southward

30 (equatorward) MSTIDs are observed almost every day during 0800-2100 LT in Spring

31 and Winter. At midnight, southward MSTIDs are more discernible in Summer and

32 majority of them are propagating from Japan to Taiwan. Second, northward (poleward)

33 MSTIDs are more frequently detected during 1200-2100 LT in Spring and Summer with

34 the secondary peak of occurrence between day of year (DOY) 100-140. The

35 characteristics of the MSTIDs are interpreted with additional observations from radio

36 occultation (RO) soundings of FORMOSAT-3/COSMIC as well as modeled

37 atmospheric waves from the high-resolution Whole Atmosphere Community Climate 

to the atmospheric gravity waves (AGWs).

40

41

43 (e.g. Hunsucker et al., 1982; Otsuka et al., 2013). MSTIDs were previously studied using various techniques in the middle latitudes such as all-sky airglow imagers, ionosondes, incoherent scatter radar, and total electron content (TEC) observations

51 derived from ground-based global navigation satellite system (GNSS) receivers. (e.g., Bowman, 1985, 1989; Garcia et al., 2000; Saito et al., 2002; Shiokawa et al., 2003a, 2006; Otsuka et al., 2004, 2011; Amorim et al., 2011; Medvedev et al., 2017; Takeo et al., 2017).

55 MSTIDs were previously categorized into daytime and nighttime types according 
57 hemisphere have feature of frontal alignment in east-west (E-W) direction propagating

58 in meridional north-south $(\mathrm{N}-\mathrm{S})$ direction or frontal alignment of southwest-northeast

59 (SW-NE) direction propagating in northwest-southeast (NW-SE) direction (Hernandez-

60 Pajareset al., 2012; Kotake et al., 2006; Otsuka et al., 2011). On the other hand, the

61 nighttime MSTID has a unique frontal alignment in the northwest-southeast (NW-SE)

62 direction with propagation direction in equator westward direction (Behnke et al., 1979;

63 Saito et al., 1998; Garcia et al., 2000; Shiokawa et al., 2003a; Kotake et al., 2006;

64 Tsugawa et al., 2007a; Kubota et al., 2011; Hernández-Pajares et al., 2012; Rajesh et

65 al., 2016). It is due to such a special frontal alignment, the phenomenon was originally

66 explained by Perkins instability (c.f. Perkins et al., 1973, Behnke, 1979, Garcia et al., 67 2000).

68 As the growth rate of Perkins instability is much slower than the observations,

69 previous studies suggested that the instability also requires seeding perturbation to

70 induce the instability development. Kelley and Fukao (1991) recommended that AGWs

71 could be a seeding mechanism to accelerate the Perkins instability. Huang et al. (1994)

72 suggested that AGWs play an important role in formation of the nighttime MSTIDs due

73 to the electrodynamic coupling, which was supported by follow-up observations (Miller

74 et al., 1997; Nicolls and Kelley, 2005). Meanwhile, Shiokawa et al. (2003b) calculated

75 the theoretical predicted growth of the Perkins instability. The result indicated that the 
76 Perkins instability has low growth rate alone and it takes more than 2 hours, which is

77 much longer than the observations. Recently, Chou et al. (2017, 2018), employed dense

78 GNSS observations and simulations on the study of MSTIDs driven by typhoon and

79 proposed that the severe weather driven concentric GWs (CGWs) could accelerate the

80 Perkins instability by providing additional polarization electric field perturbations

81 driven by the perturbation winds of CGWs.

Additionally, Sporadic E layer (EsL) instability is proposed as an important

83 indirect driver of the nighttime MSTIDs (Cosgrove and Tsunoda, 2004), which is

84 mainly produced by the effect of vertical wind shear in E region (Whitehead, 1961;

85 Mathews, 1998; Carrasco et al., 2007; Haldoupis, 2011; Yeh et al., 2013). EsL

86 instability can act as a vital character for accelerating growth of Perkins instability by

87 projecting the polarization electric field perturbations to the F-region. Tsunoda (2006)

88 showed the analytic results on the acceleration of the instability and Yokoyama et al.

89 (2009) showed the self-consistent coupling of EsL and F layer Perkins instabilities

90 through numerical simulations. Both studies have indicated the importance of this E

91 and F layer coupling for growth of the MSTIDs.

92 Not only occurring at mid-latitude, observational studies also depicted

93 characteristics of MSTIDs over the low-latitude regions (c.f. Shiokawa et al., 2006: Lee

94 et al., 2008; MacDougall et al., 2011; Fukushima et al., 2012; Narayanan et al., 2014; 
95 Jonah et al., 2016; Paulino et al., 2016; Figueiredo et al., 2018). For instance, the

96 investigations using OI $630.0 \mathrm{~nm}$ airglow images taken by all-sky imager around the

97 transition region of low to mid-latitudes $\left(19.3^{\circ} \mathrm{N}\right.$ dip latitude) reported by Narayanan et

98 al. (2014) suggested that the MSTID could be inhibited by the equatorial ionospheric

99 anomaly. Shiokawa et al. (2006) and Fukushima et al. (2012) suggested that the

100 directionalities of MSTIDs over Kototabang (10.4 ${ }^{\circ} \mathrm{S}$ dip latitude) are different from the

101 typical MSTIDs observed at the mid-latitudes and attribute their formation by gravity

102 waves. Paulino et al. (2016) carried out an investigation at the off-equatorial region,

103 over São João do Cariri, by using airglow observations over nearly a solar cycle and

104 speculated that the gravity waves are the likely source of the periodic MSTIDs.

105 As described above, MSTIDs were statistically studied in the ionosphere for quite

106 a long time in mid-latitude region with some additional documentations over low-

107 latitude region possibly driven by various physical mechanisms. Most of the studies,

108 however, were performed mainly by adopting visual inspection of the observation data

109 that requires time consuming efforts and could be influenced by subjective bias while

110 inspecting the airglow images or TEC maps. Takeo et al. (2017) performed three-

111 dimensional fast Fourier transform (3-D FFT) to detect the MSTIDs automatically in

112 airglow imagers over a solar cycle for the first time. Here, our main goal is to develop

113 an autonomous detection algorithm by combining the 3-D FFT and support vector 
114 machine (SVM) to distinguish and categorize MSTIDs from other waves or

115 irregularities in the GNSS-TEC maps.

116 In this study, the autonomously identified MSTIDs at low to mid-latitudes are

117 statistically organized according to their wave parameters, i.e. wavelength, amplitude,

118 propagation direction, seasonal and local time (LT) dependences. The possible

119 mechanisms responsible for these low-latitude MSTIDs are discussed based on these

120 statistical results with aids from the EsL observation obtained from the radio occultation

121 (RO) observations of FORMOSAT-3/COSMIC (Yeh et al., 2014) and the AGWs

122 simulated by the high-resolution Whole Atmosphere Community Climate Model

123 (WACCM) (Liu et al., 2014).

124

125 2.Methodology

126 We develop an autonomous algorithm to detect MSTIDs by using both the 3-D

127 FFT and the SVM. As the equatorial plasma bubbles (EPBs) affected by zonal neutral

128 winds could appear in the reverse C-shape spatial distribution (Shiokawa et al. 2004;

129 Kil et al., 2009) showing NW-SE (NE-SW) waveform in the northern (southern)

130 hemisphere similar to the frontal alignment of MSTIDs, it is important to have the

131 algorithm capable of distinguishing the two different instabilities. The algorithm is

132 applied on the GNSS data around Taiwan $\left(20-30^{\circ} \mathrm{N}, 115-125^{\circ} \mathrm{E}\right.$ geographic 
133 latitude/longitude and $15^{\circ} \mathrm{N}$ dip latitude) under the northern EIA crest during 2013-

1342015 to study the characteristics, e.g. propagation direction, LT and seasonal

135 dependence, of MSTIDs and EPBs.

136 Before carrying out the 3-D FFT and SVM, a 60 mins. high pass filter is applied

137 to the GNSS-TEC data followed by interpolation of the filtered TECs into the fixed

138 grids with spatial resolution of $\sim 0.5 \mathrm{deg}$. latitude/longitude in both zonal and meridional

139 direction with an accumulation period of 30 mins. It should be noted that we substitute

140 zero to the region with no available data owing to the limited coverage of GNSS

141 observation around Taiwan. The ionospheric pierce point (IPP) for converting slant to

142 vertical TEC is set at $300 \mathrm{~km}$ altitude. Figure 1a shows an example of the filtered TEC

143 over the region and Figure $1 \mathrm{~b}$ illustrates the grids for the autonomous detection

144 algorithm.

145 We exploit the 3-D FFT method similar to that developed by Matsuda et al. (2014)

146 to the cumulative three-dimensional filtered TEC data for the zonal and meridional

147 wave numbers and angular frequency. It is noted that positive wave number represents

148 northward or eastward direction of propagation. The phase velocities $\left(\mathrm{V}_{p}\right)$ of the waves

149 are given in terms of the parameters obtained from the 3-D FFT as follows,

$151 \quad \mathrm{~V}_{p}=\sqrt{\left(\frac{\omega}{2 \pi} \frac{W_{m}}{K}\right)^{2}+\left(\frac{\omega}{2 \pi} \frac{W_{z}}{L}\right)^{2}}$ 
153 where $\mathrm{W}_{\mathrm{m}}$ and $\mathrm{W}_{\mathrm{z}}$ are meridional and zonal width of the detection region; $\mathrm{K}, \mathrm{L}$ and $\omega$

154 represent meridional wave numbers, zonal wave numbers and angular frequency,

155 respectively. The propagation direction $(\theta)$ is given as,

$157 \quad \theta=\varnothing+\tan ^{-1} \frac{\left(\frac{W_{m}}{L}\right)^{2}}{\left(\frac{W_{Z}}{K}\right)^{2}}$

159 where $\varnothing$ is the azimuth angle, which depends on the value of both zonal and 160 meridional wave numbers.

The 3-D FFT will provide a weaker artificial enhancement of power spectral density (PSD) in the opposite direction than that of the true direction (Takeo et al., 2017).

163 Therefore, the maximum PSD is taken to determine the direction for each computation

164 to rule out the wrong directions in this study. of parameters including horizontal wavelength, meridional and zonal wave numbers,

167 phase velocity, propagation direction, angular frequency, PSD of the 3-D FFT,

168 minimum TEC, day of year (DOY) and universal time (UT). These parameters are

169 exploited for training the SVM model in order to distinguish MSTID, EPB and quiet time background ionosphere. The detailed procedures for creating the SVM model is 
171 illustrated in Figure 2. In the SVM model, we create a ten-dimensional space where the

172 coordinates stem from the 10 kinds of parameters mentioned above. The data are

173 represented as points and are mapped into the 10-dimensional (10-D) space according

174 to the corresponding values of each parameter. In the 10-D space, the SVM model

175 separates different classes by computing the best boundaries called hyperplanes. New

176 data are then mapped into that same space and are predicted into various classes based

177 on the side of the hyperplane on which they might fall. Presumably, the hyperplane

178 between anisotropy classes should be as wide as it could be to maintain the quality of

179 the SVM model. Therefore, a soft-margin method, defining the width of a hyperplane

180 in non-linear classification, is applied on supporting the hyperplane. We define a

181 hyperplane with a soft-margin in the following form (Chen et al., 2004; Chang et al,

182 2011),

183

$184\left|N_{h} \cdot \mathrm{x}-\mathrm{b}\right| \geq 1 \pm \varepsilon_{i}$

186 where $N_{h}$ represents the normal vector to the hyperplane; x refers to the normalized

187 10-D parameters; $b$ is a normal constant; the normal constant (on the right-hand side of

188 the equation) "1" or "-1", considering the absolute value, indicates two different classes;

$189 \varepsilon_{i}$ represents slack variable which determine the width of the soft-margin. It should be 
apparent that the greater the value of slack variable is, the larger the breadth of the soft-

191 margin would be. In order to maintain the quality of classification, the slack variable

192 should as small as it could be where we set the value as 0.4 in our case.

In this study, a supervised learning method is used for classifying the different

194 classes. Supervised learning indicates that we have to label the classes for the raw data

195 to create input-output pairs before training the SVM model. In our case, we label the

196 different classes using discriminants and a little visual inspection for validation. During

197 the training processes, a solar maximum year, 2000, and a solar minimum year, 2009,

198 are used for training and testing the model quality since MSTIDs and EPBs have a

199 different solar activity dependence. Due to the 30 mins time resolution in the 3D-FFT

200 computation, the total number of data for these two years are around 35000 . The dataset

201 is randomly separated into two groups including $80 \%$ of training data ( 28000 piece)

202 and the other $20 \%$ of testing data ( $~ 7000$ piece). The training data are used for training

203 the model by computing a hyperplane for categorizing anisotropy classes. On the other

204 hand, the testing data applied on testing the trained model are utilized for calculating

205 the accuracy rate of the SVM model prediction. Here, the accuracy is the ratio of

206 number of correct predictions to the total number of input samples. During the testing

207 processes, the dataset is repeatedly split into training data and testing data for five times 
to test the model performance. The testing results indicate that our model has over $96 \%$ accuracy rate for distinguishing MSTID, EPB and ionosphere without irregularity.

\section{Results and Discussions}

216 November to 6 February for 3 months, centering on the winter solstice.

220 dependence. Here, the occurrence rate is defined as the ratio between the number of

221 events in which EPBs are observed (M) and the amount of data in which GNSS-TEC

222 exist (N). Since the GNSS-TEC data are available every 30 seconds during 2013-2015,

223 the value of $\mathrm{N}$ is identical in each occurrence rate computation.

225 follows (figure not shown). First, the EPBs are usually observed at the LT period of 
227 is higher in the solar maximum year of 2014, showing the dependence on the solar

228 activity. Third, the cumulative occurrence rate for these years indicates that the seasonal

229 occurrence rate of EPBs is descending in the order from Spring, Fall, Summer, to Winter.

230 The characteristics of EPBs in their diurnal and seasonal variations are consistent with

231 those reported in the previous studies (e.g. Sahai et al., 2000; Huang et al., 2002;

232 Pimenta et al., 2003; Fejer et al., 2005; Gentile et al., 2006; Yao \& Makela, 2007;

233 Paulino et al., 2011; Sharma et al., 2014; Sun. et al., 2016) suggesting the reliability of

234 our algorithm.

235

\subsection{Observation Results}

In this section, we display the annual variations (2013-2015) of the characteristics

238 of MSTIDs by excluding the possible contamination coming from EPBs. The

239 wavelength and horizontal phase velocity characteristic of MSTIDs over Taiwan are

240 respectively among 100-400 $\mathrm{km}$ and $100-250 \mathrm{~m} / \mathrm{s}$ which are consistent with the

241 previous studies (e.g. Hunsucker et al., 1982; Otsuka et al., 2013). The occurrence rate

242 of MSTIDs are calculated by using the same approach applied for calculation of the

243 occurrence of EPBs described in Section 3. The results illustrate that the occurrence

244 and propagation characteristics of MSTIDs have clear seasonal and LT dependences.

245 The occurrence rate of southward MSTIDs (Figure 3a-c) indicates that they are more 
246 discernable during 0800-2100 LT in Spring and Winter. During Summer, southward

247 MSTIDs mainly occur around 2100-0300 LT. On the contrary, the occurrence rate of

248 northward MSTIDs (Figure 3d-3f) demonstrates that they are more frequently observed

249 around 1200-2100 LT from Spring to Fall with a secondary peak occurring around

250 0000-0300 LT between DOY 100-140. In Figure 3g-3i, a transition boundary between

251 the northward and southwestward MSTIDs takes place three hours after sunset at

252 around 2100 LT during Summer. It is noteworthy that the LT dependence of the

253 southwestward MSTIDs takes place much later after sunset than those reported in the

254 previous literatures (Behnke et al., 1979; Saito et al., 1998; Garcia et al., 2000;

255 Shiokawa et al., 2003a; Kotake et al., 2006; Tsugawa et al., 2007a; Kubota et al., 2011;

256 Hernández-Pajares et al., 2012; Otsuka et al., 2013), which suggests an alternative

257 driving mechanism should be proposed. A rational explanation is that the

258 southwestward MSTIDs are mainly propagating from higher latitude to the lower

259 latitude over Taiwan region instead of being generated locally.

260 The filtered TEC over Japan and Taiwan (Figure 4a-4b) reveals the relationship

261 between the MSTIDs at low and mid latitudes. To identify the characteristics of the

262 MSTIDs over Japan and Taiwan, we organize filtered TEC as a function of distances

263 and time (Figure 4c). The filtered TEC along the orange dash line in Figure $4 \mathrm{~b}$ is chosen

264 for organizing Figure $4 c$ for studying the characteristics of MSTIDs in this case. The 
265 daytime MSTIDs (Figure 4a) propagate toward southeast and northwest over Japan and

266 Taiwan, respectively. On the contrary, the nighttime MSTIDs (Figure 4b) demonstrate

267 that they are propagating southwestward over Japan and across a long distance to reach

268 Taiwan region. In Figure 4c, results apparently show that the MSTIDs over Japan have

269 greater wave amplitudes $\left(\sim 0.2 \mathrm{TECu}\right.$, where $\left.1 \mathrm{TECu}=10^{16} \mathrm{el} / \mathrm{m}^{2}\right)$ and longer

270 wavelengths $(\sim 250 \mathrm{~km})$ than those over Taiwan where the wave amplitude is around

$2710.1 \mathrm{TECu}$ and the wavelength is about $100 \mathrm{~km}$. The red rectangle in Figure $4 \mathrm{c}$, therefore,

272 illustrates that the MSTIDs around 1400-2100 UT (2200-0500 LT) over $22-28^{\circ} \mathrm{N}$

273 latitude are mainly coming from Japan as they have a greater TEC amplitude fluctuation

274 and wavelengths than those in the same latitudes at earlier local times.

275 To further identify the relationship between the MSTIDs over Japan and Taiwan,

276 we compare the propagation direction with normalized power spectral density (PSD) of

277 MSTIDs (Figure 5) between DOY 100-300 during 2013-2015. The PSD derived from

278 3-D FFT indicates the power of the waves, which corresponds to the square of the

279 amplitude fluctuation. The normalized PSD with greater value, indicating perturbations

280 with greater wave amplitude, mainly occur three hours after sunset. This is in good

281 agreement with the southwestward MSTID observations, further suggesting that these

282 MSTIDs are mainly propagating from Japan instead of being generated over Taiwan. 


\subsection{Perkins /Es Layer Instability and MSTIDs}

the most plausible generation mechanism to explain the special wavefront alignment

(NW-SE) of MSTIDs (Benke, 1979; Garcia et al., 2000). According to Shiokawa et al.

given as:

290

$$
\begin{aligned}
\gamma_{p} & =\frac{g}{H_{n}<v_{\text {in }}>} \frac{\sin ^{2}(I) \sin (\theta-\alpha) \sin (\alpha)}{\cos (\theta)} \\
& =\frac{E_{0} \sin (\theta-\alpha) \sin (\alpha) \cos (I)}{B H_{n}} \frac{<v_{\text {in }}>_{o t}}{<v_{\text {in }}>}
\end{aligned}
$$

295 where $\mathrm{g}, H_{n}$ and $v_{\text {in }}$ are the gravitational acceleration, scale height of neutral

296 atmosphere and ion-neutral collision frequency; I represents the magnetic inclination;

$297 \theta$ is the angle of background electric field from east; $\alpha$ means the angle between the

298 direction normal to the frontal structure and east; $E_{0}, \mathrm{~B}$ and are the background electric

299 field and magnetic field; subscript 0t represents inclusion of a tangential component.

300 Notice that $\alpha$ should greater than zero but less than or equal to $\theta$.

In Equation (4), the ion-neutral collision frequency is inversely proportional to the 
303 of solar activity, which generates a lower ion-neutral collision frequency. This effect

304 could produce a high occurrence rate of nighttime MSTIDs during solstices and low

305 solar flux years which agree with the previous literatures (e.g. Shiokawa et al., 2003a;

306 Kotake et al., 2006).

307

On the other hand, a coupled electrodynamical system between EsL and F region

308 also plays an important role to accelerate the growth rate for nighttime MSTIDs (e.g.

309 Cosgrove \& Tsunoda, 2004; Tsunoda, 2006; Yokoyama et al., 2009). In this study, we

310 estimate the occurrence of EsL by using FORMOSAT-3/COSMIC (F3/C) radio

311 occultation (RO) data for comparing with the MSTIDs characteristics in nighttime

312 during 2013-2015. Resende et al. (2018) revealed that the S4 scintillation index derived

313 from the $\mathrm{F} 3 / \mathrm{C} \mathrm{RO}$ profiles, which provide the disturbance information from the signal

314 to noise ratio (SNR) of the electron density from GPS L1 band, is suitable to estimate

315 the EsL at low latitudes due to their high correlation. In this case, an S4 threshold of 0.3

316 is set over 80-130 km altitudes (c.f. Whitehead, 1989; Chu et al., 2014; Yeh et al., 2014;

317 Tsai et al., 2018) for evaluating the occurrence of EsL. It is noteworthy that the EsL do

318 not have a conjugate effect but the MSTIDs are presented as conjugate structures in

319 both hemispheres according to previous studies (Saito et al., 1998; Otsuka et al., 2004;

320 Martinis et al., 2019). That is, the EsL occurring at one of the hemispheres could trigger

321 the MSTID in both hemispheres, coherently. As a result, two detection regions are 
322 formed, which are (i) Taiwan region, the same as mentioned in Section 2, and (ii) the

323 conjugate region of Taiwan, the identical meridional and zonal breadth to (i) but

324 centering at $120^{\circ} \mathrm{E}$ geomagnetic longitude; $-4^{\circ} \mathrm{S}$ geomagnetic latitude, for investigating

325 the EsL characteristics in nighttime during 2013-2015.

326 The EsL mainly occur in Summer but the occurrence is much less over Taiwan

327 (Figure 6a) than over Japan (Otsuka et al., 2008) in nighttime, which is consistent with

328 the previous study (Zhou et al., 2017). On the contrary, the southward/northward

329 (Figure 6c/6d) MSTIDs still have considerable occurrence rate until 2200 LT in Spring

330 and Winter/2100 LT in Spring to Fall with the identical propagation direction (Figure

$3316 b$ ) before and after the dusk terminator (black line). Also, the propagation direction of

332 the northward MSTIDs (Figure 7) illustrates that the MSTIDs mainly propagate

333 northwestward (wavefront of NE-SW direction) and exactly northward (wavefront of

334 E-W direction), which are inconsistent with the theoretical wavefront alignment (NW-

335 SE) of Perkins instability and EsL instability (Perkins, 1973; Cosgrove and Tsunoda,

336 2004). The anisotropy characteristics between EsL and MSTIDs rules out the possibility

337 that the Perkins instability and EsL instability are responsible for generating the 338 nighttime MSTIDs over Taiwan. 
342 and propagation, AGWs are considered as a plausible driver of the northward-

343 propagating MSTIDs in Spring and Summer nighttime over Taiwan. The secondary

344 peak of occurrence of northward MSTIDs taking place post-midnight between DOY

345 100-140 should be amenable to interpretation in terms of AGWs. Figure 8 displays an

346 example of $557 \mathrm{~nm}$ airglow images on 29 April 2020 around 0209-0239 LT, obtained

347 from an all sky imager operating at Tainan Astronomical Education Area $\left(120.39^{\circ} \mathrm{E}\right.$;

$\left.34823.18^{\circ} \mathrm{N}\right)$. The images, mainly contributed from about $95-97 \mathrm{~km}$ altitude, are used to

349 evaluate the horizontal structures of AGWs during this period. Using the 3-D FFT, the

350 series of airglow images demonstrate the salient northeastward propagation of AGWs

351 with a horizontal phase velocity, horizontal wavelength, and period of $\sim 114 \mathrm{~m} / \mathrm{s}, \sim 83$

$352 \mathrm{~km}$, and $\sim 12 \mathrm{~min}$. respectively over Taiwan. Meanwhile, the filtered TEC map with an

353 IPP at $300 \mathrm{~km}$ altitude (Figure 9) reveals the northeastward MSTIDs with a horizontal

354 phase velocity, horizontal wavelength, and period of $\sim 147 \mathrm{~m} / \mathrm{s}, \sim 151 \mathrm{~km}$, and $\sim 17 \mathrm{~min}$.,

355 respectively. The nearly identical propagation direction and similar characteristics of

356 AGWs and MSTIDs suggesting that AGWs should be responsible for the nighttime

357 MSTIDs over Taiwan for this event. However, the time characteristic in the airglow

358 images and filtered TEC maps are different in this case suggesting that further

359 correlation between each other should be proposed. 
361 the resolved AGWs derived from high-resolution WACCM among 30 June to 11 July.

362 In the high resolution WACCM, the horizontal and vertical resolution are $\sim 0.25^{\circ}$ and

$363 \sim 700 \mathrm{~m}$, respectively. In this case, we investigate AGWs horizontal structure at 5.8e-

364 4hpa ( 93 km) altitude (c.f. Zhou et al., 2002; Suzuki et al., 2007; Chun and Kim, 2008;

365 Heale et al., 2020) in the WACCM. Taking 1400 UT on 9 July for example, the zonal

366 wind (Figure 10a) and meridional wind (Figure 10b) patterns display several salient

367 northwestward AGWs structures propagating over north Pacific Ocean and across a

368 long distance $\sim 2800 \mathrm{~km}$ along the Philippines, before reaching Taiwan. In this case, the

369 waves structures mostly dissipate around $30^{\circ} \mathrm{N}$ geographic latitude by the stronger in

370 phase background wind. In Figure 10c-10d, we estimate the morphological

371 characteristics of AGWs as a function of time and distances by organizing the zonal and

372 meridional wind along the yellow arrow in Figure 10a and 10b. Several long-lasting,

$373 \sim 12 \mathrm{hr}$, northwestward AGWs are found during this period with a horizontal wavelength

374 of tens to hundreds of $\mathrm{km}$ and a horizontal phase velocity among $20-85 \mathrm{~m} / \mathrm{s}$ which are

375 consistent with previous literatures (Piani et al., 2000; Horinouchi et al., 2002; Sentman

376 et al., 2003; Suzuki et al., 2007; Takeo et al., 2017; Tsuchiya et al., 2019).

377 In Figure 11, the propagation direction characteristic of AGWs (red line with stars),

378 obtained from the 3-D FFT over Taiwan, is compared to the MSTID observations (blue 
379 line) in Summer during 2013-2015. The locally generated MSTIDs mainly occurring

380 during 1200-2100 LT in Summer (Figure 3) are in good agreement with the

381 corresponding WACCM output suggesting that AGWs may play a role in the generation

382 of MSTIDs during this period. However, there is a clear disagreement occurring after

3832000 LT showing southwestward propagation of MSTIDs while AGWs are northward.

384 The disagreement confirms that the southwestward MSTIDs after 2100 LT mainly come

385 from the southwestward propagation of MSTIDs originated from Japan as described in

$386 \quad$ Section 3.1.

387 Tsugawa et al (2007a) suggested that the MSTID activity over Japan is highest in

388 the nighttime during 2100-0300 JST in Summer (May-August or DOY 140-250). Since

389 the amplitude of the MSTIDs over Japan is greater than that over Taiwan, it is difficult

390 to isolate any influence of Japan MSTID's in the overlapping TEC observations over

391 Taiwan. However, the DOY from 100-140, when the southwestward MSTIDs over

392 Japan were least observed could be the opportunity for the identification of locally

393 generated MSTIDs over Taiwan. This characteristic of MSTIDs, when they are present,

394 is consistent with a secondary occurrence peak of the northward MSTIDs over Taiwan

395 suggesting that this secondary peak of northward MSTIDs could be identified owing to

396 the least occurrence of MSTIDs over Japan during the period. Also, the propagation

397 direction characteristic of AGWs after 2000 LT (Figure 11) is mainly propagating 
northwestward which is consistent with the secondary peak of the MSTIDs generation of the northward-propagating MSTIDs over Taiwan in nighttime in Summer.

401 Based on the propagation directions and seasonal occurrences, we suggest that the 402 AGWs have a great contribution to the low latitude MSTIDs in both daytime and 403 nighttime in Summer.

\section{Discussion} alternative explanation is that they are mainly coming from mid-latitude region. AGWs seeding, however, could be a plausible mechanism to accelerate the Perkins instability where the growth of the instability could be significantly enhanced and accelerated

410 (Kelly and Fukao, 1991; Huang et al., 1994; Chou et al., 2017). Such a mechanism,

411 hence, indicates that the southwestward MSTIDs could be generated locally due to the

412 Perkins instability as well. In order to understand further about the generation 413 mechanism of the southwestward MSTIDs, the occurrence rate of southwestward 414 MSTIDs are compared between Japan and Taiwan. Otsuka et al. (2011) revealed that 415 the occurrence rate is $\sim 30 \%$ over Japan in a solar maximum year in nighttime in 416 Summer. Our study, however, shows the higher occurrence rates of $\sim 40 \%$ in a solar 
417

418

419

420

421 et al. (2011) (f10.7 index is 179.4 s.f.u.) suggesting that the further relationship between

422 the MSTIDs over Japan and Taiwan should be investigated in the future works.

$424 \quad 5$. Summary

425 In this study, we utilize both the 3-D FFT and SVM to statistically investigate the

426 MSTIDs in the low latitude equatorial ionization anomaly region over Taiwan during

427 2013-2015. As EPBs have some characteristics similar to MSTIDs, the occurrence of

428 EPBs is also investigated in order to distinguish it from the MSTIDs. Several important

429 features such as the variation of the propagation direction and occurrence rates are

430 revealed in this study. The main findings are summarized as follows.

1. The statistical results show that the seasonal, LT variations and solar activity dependence are generally consistent to previous studies of MSTIDs and EPBs, indicating that our algorithms could successfully distinguish EPBs and MSTIDs in the TEC perturbations. 
2. The occurrence rate and propagation direction of MSTIDs have clear seasonal and LT dependences. In Spring and Winter, southward MSTIDs are observed almost every day during 0800-2100 LT, and in Summer they appear mainly during 2100-0300 LT and have least occurrence in Autumn. On the contrary, northward MSTIDs are observed more frequently during 1200-2100 LT from Spring to Autumn with a secondary peak during 0000-0300 LT between DOY 100-140.

3. The propagation directions of MSTIDs patterns display a clear boundary for northward and southwestward MSTIDs at 2100 LT in Summer. During 12002100 LT, the MSTIDs mainly propagate northward and northwestward. On the other hand, the MSTIDs observed during 2100-0300 LT mainly propagating southwestward are majorly coming from Japan, mid-latitude region. that the MSTID in Taiwan region are influenced by the southwestwardpropagating MSTIDs from Japan during 2100-0300 LT between DOY 140-250 northward MSTIDs during 0000-0300 LT between DOY 100-140, therefore, could be generated owing to the least occurrence of MSTIDs over Japan. 
5. The wavefront alignment characteristic of nighttime MSTIDs is inconsistent with the Perkins and EsL instability, ruling out the possibility of these generation mechanisms. In contrast, the relationship between $\lambda 557 \mathrm{~nm}$ airglow images and filtered TEC map together with a simulation result from WACCM illustrate that AGWs.

461

462 Declarations

$464 \quad$ Not applicable.

Not applicable.

AGWs : atmospheric gravity waves.

469 CGWs : concentric gravity waves.

DOY : day of year.

471 EIA : equatorial ionization anomaly. EPBs : equatorial plasma bubbles. 
EsL : sporadic E layer.

F3/C : FORMOSAT-3/COSMIC.

GNSS : global navigation satellite system.

476

IPP : ionospheric pierce point.

477

LT : local time.

478

MSTIDs : medium-scale traveling ionospheric disturbances.

479

PSD : power spectral density.

480

$\mathrm{RO}$ : radio occultation.

481

SNR : signal to noise ratio.

482

SVM : support vector machine.

483

S4 : scintillation index.

484

TEC : total electron content.

485

TIDs : traveling ionospheric disturbances.

486

$\mathrm{UT}:$ universal time.

487

WACCM : Whole Atmosphere Community Climate Model.

488

3-D FFT : three-dimensional fast Fourier transform. 
The GNSS-TEC data are provided by the Central Weather Bureau in Taiwan (https://gdms.cwb.gov.tw/) and Geospatial Information Authority in Japan (http://www.gsi.go.jp/ENGLISH/index.html). The F3/C data are provided by Taiwan Analysis Center for COSMIC (TACC) (https://tacc.cwb.gov.tw/v2/). The all sky imagers data are available on the all sky observatory (ALSO) (http://allskyairglow.earth.ncku.edu.tw/PicWeb/MainHTML/2020/04/29). The f10.7 index is NASA (https://omniweb.gsfc.nasa.gov/).

\section{Competing interests}

499 The authors declare that they have no competing interests.

\section{Funding}

501 This paper is supported by Ministry of Science and Technology (MOST) of Taiwan to National Cheng Kung University under the project MOST 108-2638-M-006-

503 001-MY2.

\section{Authors' contributions}

PHC drafted the manuscript, created the SVM model, carried out 3D-FFT method, program coding and analyzed the data. CHL and PKR critically evaluated the text for scientific content and elaborated it. CHL and YO gave very important conceptions on analyzing the observational results. HLL dealt with the WACCM. 

authors read and approved the final manuscript.

\section{Acknowledgements} and ALSO provide the data. Also, thanks to the MOST supports the funding.

\section{References}

Amorim, D. C. M., Pimenta, A. A., Bittencourt, J. A., \& Fagundes, P. R. (2011). Long term study of medium-scale traveling ionospheric disturbances using oi $630 \mathrm{~nm}$ Geophysical Research, 116, A06312. doi.org/10.1029/2010JA016090 climatology of ionospheric irregularities derived from GPS radio occultation, Geophys. Res. Lett., 35, L14809, doi:10.1029/2008GL034158.

523 Arras, C., C. Jacobi, and J. Wickert (2009): Semidiurnal tidal signature in sporadic E midlatitudes, Ann. Geophys., 27, 2555-2563.

526 Behnke, R. (1979), F layer height bands in the nocturnal ionosphere over Arecibo, J. 
528 Bowman, G. G. (1985). Some aspects of mid-latitude spread-Es and its relationship with spread-F. Planetary and Space Science, 33(9), 1081-1089. doi.org/10.1016/0032-0633(85)90027-3

531 Bowman, G. G., Quasi-periodic scintillations at mid-latitudes and their possible association with ionospheric sporadic-E structures, Ann. Geophys., 7, 259, 1989.

533

534

535

536

537

538

539

540

541

542

Burges, C. J. (1998). A tutorial on support vector machines for pattern recognition. Data mining and knowledge discovery, 2(2), 121-167.

Carrasco, A. J., I. S. Batista, and M. A. Abdu, Simulation of the sporadic E layer response to prereversal associated evening vertical electric field enhancement near dip equator, J. Geophys. Res., 112, A06324, doi:10.10292006JA01243, 2007.

C.-C. Chang and C.-J. Lin. LIBSVM: a library for support vector machines, 2001. Software available at http://www.csie.ntu.edu.tw/ cjlin/libsvm.

D. R. Chen, Q. Wu, Y. Ying, and D.-X. Zhou, Support vector machine soft margin classifiers: Error analysis, J. Mach. Learn. Res. 5 (2004), 1143-1175.

Chou, M. Y., Lin, C. C., Yue, J., Chang, L. C., Tsai, H. F., \& Chen, C. H. (2017). Medium-scale traveling ionospheric disturbances triggered by Super Typhoon Nepartak (2016). Geophysical Research Letters, 44(15), 7569-7577.

Chou, M. Y., C. H. Lin, J. D. Huba, Lein, C. P., C. H. Chen, J. Yue, L. C. Chang and P. K. Rajesh (2018), Numerical modeling of the concentric gravity wave seeding of 

low-latitude nighttime medium-scale traveling ionospheric disturbances, Geophysical Research Letters, 45. https://doi.org/10.1029/2018GL077959

549 Chun, H. Y., \& Kim, Y. H. (2008). Secondary waves generated by breaking of 550 convective gravity waves in the mesosphere and their influence in the wave momentum flux. Journal of Geophysical Research: Atmospheres, 113(D23).

552 Cooley, J. W., \& Tukey, J. W. (1965). An algorithm for the machine calculation of 553 complex Fourier series. Mathematics of computation, 19(90), 297-301.

554 Cosgrove, R. B., and R. T. Tsunoda (2004), Instability of the E-F coupled nighttime 555 midlatitude ionosphere, J. Geophys. Res., 109, A04305, doi:10.1029/2003JA010243.

557 Chu, Y. H., Wang, C. Y., Wu, K. H., Chen, K. T., Tzeng, K. J., Su, C. L., ... \& Plane, J. 558 M. C. (2014). Morphology of sporadic E layer retrieved from COSMIC GPS radio

561 Danielson, Gordon C.; Lanczos, Cornelius (1942). "Some improvements in practical 562 Fourier analysis and their application to x-ray scattering from liquids". Journal of 563 the Franklin Institute. 233 (4): 365-380. 
564

565

566

567

568

569

570

571

572

573

574

575

576

577 Fukushima, D., K. Shiokawa, Y. Otsuka, and T. Ogawa (2012), Observation of

Farley, D. T., Bonelli, E., Fejer, B. G., and Larsen, M. F. (1986). The prereversal enhancement of the zonal electric field in the equatorial ionosphere. J. Geophys. Res. $91,13,723$.

Fejer, B. G., Souza, J. R., Santos, A. S., \& Costa Pereira, A. E. (2005). Climatology of F region zonal plasma drifts over Jicamarca. Journal of Geophysical Research, 110, A12310.

Figueiredo, C. A. O. B., Takahashi, H., Wrasse, C. M., Otsuka, Y., Shiokawa, K., \& Barros, D. (2018). Medium-scale traveling ionospheric disturbances observed by detrended total electron content maps over Brazil. Journal of Geophysical Research: Space Physics, 123(3), 2215-2227.

578

579 airglow images over 7 years, J. Geophys. Res., 117, A10324, doi:10.1029/2012JA017758. 
581 Garcia, F. J., M. C. Kelley, J. J. Makela, and C.-S. Huang (2000), Airglow observations of mesoscale low-velocity traveling ionospheric disturbances at midlatitudes, J. Geophys. Res., 105(A8), 18,407-18,415, doi:10.1029/1999JA000305.

584 Gentile, L. C., Burke, W. J., \& Rich, F. J. (2006). A climatology of equatorial plasma 585 bubbles from DMSP 1989-2004. Radio Science, 41(5).

586 Haldoupis C. 2011. A tutorial review on sporadic E layers. In Aeronomy of the Earth's atmosphere and ionosphere (eds A Bhattacharyya, MA Abdu, D Pancheva), pp. 381-394. Dordrecht, The Netherlands: Springer.

589 Heale, C., Bossert, K., Vadas, S. L., Hoffmann, L., Dörnbrack, A., Stober, G., Snively, 590 J. B., \& Jacobi, C. (2020). Secondary gravity waves generated by breaking mountain waves over Europe. Journal of Geophysical Research: Atmospheres, 125, e2019JD031662. https://doi.org/10.1029/2019JD031662

593 Hernández-Pajares, M., Juan, J. M., Sanz, J., \& Aragón-Àngel, A. (2012). Propagation 594 of medium scale traveling ionospheric disturbances at different latitudes and solar 595 cycle conditions. Radio Science, 47(06), 1-22.

596 Hines, C. O. (1960), Internal atmospheric gravity waves at ionospheric heights, Can. J. Phys., 38, 1441 
598 Horinouchi, T., T. Nakamura, and J. Kosaka (2002), Convectively generated mesoscale

599 gravity waves simulated throughout the middle atmosphere, Geophys. Res. Lett., 600 29(21), 2007, doi:10.1029/2002GL016069.

601 Huang, C. Y., Burke, W. H., Machuzak, J. S., Gentile, L. C., \& Sultan, P. J. (2002).

602 Equatorial plasma bubbles observed by DMSP satellites during a full solar cycle:

603 Toward a global climatology. Journal of Geophysics, 107(A12), 1434.

604 Hunsucker RD (1982) Atmospheric gravity waves generated in the high-latitude

605 ionosphere: a review. Rev Geophys Space Phys 20(2):293-315.

606 doi:10.1029/RG020i002p00293

607 Jonah, O. F., Kherani, E. A., \& De Paula, E. R. (2016). Observation of TEC perturbation

608 associated with medium-scale traveling ionospheric disturbance and possible

609 seeding mechanism of atmospheric gravity wave at a Brazilian sector. Journal of

610 Geophysical Research: Space Physics, 121(3), 2531-2546.

611 Kil, H., Paxton, L. J., \& Oh, S.-J. (2009). Global bubble distribution seen from

612 ROCSAT-1 and its association with the pre-reversal enhancement. Journal of

613 Geophysical Research, 114, A06307. doi.org/10.1029/2008JA013672

614 Kotake, N., Y. Otsuka, T. Tsugawa, T. Ogawa, and A. Saito (2006), Climatological study

615 of GPS total electron content variations caused by medium-scale traveling 
ionospheric disturbances, J. Geophys. Res., 111, A04306, doi:10.1029/2005JA011418.

618

619

620

621

622

623

624

625

626

627

628

629

630

631

632

633

634

Kotake, N., Otsuka, Y., Ogawa, T., Tsugawa, T., \& Saito, A. (2007). Statistical study of medium-scale traveling ionospheric disturbances observed with the GPS networks in Southern California. Earth, planets and space, 59(2), 95-102.

Kubota, M., M. Conde, M. Ishii, Y. Murayama, and H. Jin (2011), Characteristics of nighttime medium-scale traveling ionospheric disturbances observed over Alaska, J. Geophys. Res., 116, A05307, doi:10.1029/2010JA016212.

Lakshmi Narayanan, V., Shiokawa, K., Otsuka, Y., \& Saito, S. (2014). Airglow observations of nighttime medium-scale traveling ionospheric disturbances from Yonaguni: Statistical characteristics and low-latitude limit. Journal of Geophysical Research: Space Physics, 119(11), 9268-9282.

Lee, C. C., Y. A. Liou, Y. Otsuka, F. D. Chu, T. K. Yeh, K. Hoshinoo, and K. Matunaga (2008), Nighttime medium-scale traveling ionospheric disturbances detected by network GPS receivers in Taiwan, J. Geophys. Res., 113, A12316, doi:10.1029/2008JA013250.

Liu, H. L., McInerney, J. M., Santos, S., Lauritzen, P. H., Taylor, M. A., \& Pedatella, N. M. (2014). Gravity waves simulated by high-resolution whole atmosphere community climate model. Geophysical Research Letters, 41(24), 9106-9112. 
635 636 637 638 639 640 641 642 643 644 645 646 647 648 649 650

MacDougall, J., M. A. Abdu, I. Batista, R. Buriti, A. F. Medeiros, P. T. Jayachandran, and G. Borba (2011), Spaced transmitter measurements of medium scale traveling ionospheric disturbances near the equator, Geophys. Res. Lett., 38, L16806, doi:10.1029/2011GL048598.

Martinis, C., Baumgardner, J., Mendillo, M., Wroten, J., MacDonald, T., Kosch, M., Lazzarin, M., \& Umbriaco, G. (2019). First conjugate observations of mediumscale traveling ionospheric disturbances (MSTIDs) in the Europe-Africa longitude sector. Journal of Geophysical Research: Space Physics, 124, 2213- 2222. doi.org/10.1029/2018JA026018

Mathews, J. D. (1998), Sporadic E: Current views and recent progress,J. Atmos. Sol. Terr. Phys., 60(4), 413-435, doi:10.1016/S1364-6826(97)00043-6.

Matsuda, T. S., T. Nakamura, M. K. Ejiri, M. Tsutsumi, and K. Shiokawa (2014), New statistical analysis of the horizontal phase velocity distribution of gravity waves observed by airglow imaging, J. Geophys. Res. Atmos., 119, 9707- 9718, doi:10.1002/2014JD021543.

Medvedev, A. V., Ratovsky, K. G., Tolstikov, M. V., Oinats, A. V., Alsatkin, S. S., \& Zherebtsov, G. A. (2017). Relation of internal gravity wave anisotropy with neutral wind characteristics in the upper atmosphere. Journal of Geophysical Research: Space Physics, 122, 7567-7580. doi.org/10.1002/2017JA024103 
654 Miller, C. A., W. E. Swartz, M. C. Kelley, M. Mendillo, D. Nottingham, J. Scali, and B. 655 Reinisch (1997), Electrodynamics of midlatitude spread F : 1. Observations of 656 unstable, gravity wave-induced ionospheric electric fields at tropical latitudes, J. Geophys. Res., 102, 11,521-11,532, doi:10.1029/96JA03839.

658 Lakshmi Narayanan, V., Shiokawa, K., Otsuka, Y., \& Saito, S. (2014). Airglow 659 observations of nighttime medium-scale traveling ionospheric disturbances from 660 Yonaguni: Statistical characteristics and low-latitude limit. Journal of Geophysical 661 Research: Space Physics, 119(11), 9268-9282. observations of medium-scale traveling ionospheric disturbances at midlatitude 664 using all-sky airglow imagers, Geophys. Res. Lett., 31, L15803, doi:10.1029/2004GL020262.

666 Otsuka, Y., Tani, T., Tsugawa, T., Ogawa, T., \& Saito, A. (2008). Statistical study of 667 relationship between medium-scale traveling ionospheric disturbance and 668 sporadic E layer activities in summer night over Japan. Journal of Atmospheric 669 and Solar - Terrestrial Physics, 70, 2196-2202.

670 Otsuka, Y., Kotake, N., Shiokawa, K., Ogawa, T., Tsugawa, T., \& Saito, A. (2011). 671 Statistical study of medium-scale traveling ionospheric disturbances observed 
with a GPS receiver network in Japan. In Aeronomy of the Earth's Atmosphere and

Otsuka, Y., K. Suzuki, S. Nakagawa, M. Nishioka, K. Shiokawa, and T. Tsugawa (2013), GPS observations of medium-scale traveling ionospheric disturbances over Europe, Ann. Geophys., 31,163-172,2013, doi:10.5194/angeo-31-163-2013.

677

Paulino, I., de Medeiros, A. F., Buriti, R. A., Takahashi, H., Sobral, J. H. A., \& Gobbi, D. (2011). Plasma bubble zonal drift characteristics observed by airglow images over Brazilian tropical region Brazilian. Journal of Geophysics, 29(2), 239-246. Leite, D., Filgueira, S., Bageston, J. V., Sobral, J. H. A., and Gobbi, D.: Periodic waves in the lower thermosphere observed by OI630 nm airglow images, Ann. Geophys., 34, 293-301, https://doi.org/10.5194/angeo-34-293-2016, 2016.

Perkins, F. (1973), Spread F and ionospheric currents, J. Geophys. Res., 78, 218, 685 doi:10.1029/JA078i001p00218. three-dimensional gravity waves triggerd by deep tropical convection and their role in the dynamics of the QBO, J. Atmos. Sci., 57, 3689-3702. H., \& Taylor, M. (2003). Ionospheric plasma bubble zonal drifts over the tropical 
region: A study using OI $630 \mathrm{~nm}$ emission all-sky images. Journal of Atmospheric (2016), Space-based imaging of nighttime medium-scale traveling ionospheric disturbances using FORMOSAT-2/ISUAL $630.0 \mathrm{~nm}$ airglow observations, J. Geophys. Res. Space Physics, 121, 4769-4781, doi:10.1002/2015JA022334.

697

Resende Chagas, L. C., Arras, C., Batista, I. S., Denardini, C. M., Bertollotto, T. O., \& 698 Moro, J. (2018). Study of sporadic E layers based on GPS radio occultation 699 measurements and digisonde data over the Brazilian region. In Annales Geophysicae (Vol. 36, pp. 587-593).

701 Sahai, Y., Fagundes, P. R., \& Bittencourt, J. A. (2000). Transequatorial F-region ionospheric plasma bubbles: Solar cycle Effects. Journal of Atmospheric Solar Terrestrial Physics, 62(15), 1377-1383.

Saito, A., T. Iyemori, L. G. Blomberg, M. Yamamoto, and M. Takeda (1998), Conjugate observations of the mid-latitude electric field fluctuations with the MU radar and the Freja satellite, J. Atmos. Sol. Terr. Phys., 60, 129-140. 

and a GPS network,Earth Planets Space, 54,31-44.

711

712

713

714

715

716

717

718

719

720

721

722

723

724

725

726

Sentman, D. D., E. M. Wescott, R. H. Picard, J. R. Winick, H. C. Stenbaek-Nielsen, E. M. Dewan, D. R. Moudry, F. T. São Sabbas, M. J. Heavner, and J. Morrill (2003), Simultaneous observations of mesospheric gravity waves and sprites generated by a midwestern thunderstorm, J. Atmos. Terr. Phys., 65, 537-550.

Sharma, A. K., Nade, D. P., Nikte, S. S., Patil, P. T., Ghodpage, R. N., Vhatkar, R. S., Rokade, M. V., \& Gurubaran, S. (2014). Occurrence of equatorial plasma bubble over Kolhapur. Advances in Space Research, 54, 435-442.

Shiokawa, K., C. Ihara, Y. Otsuka, and T. Ogawa (2003a), Statistical study of nighttime medium-scale traveling ionospheric disturbances using midlatitude airglow images, J. Geophys. Res.,108(A1), 1052, doi:10.1029/2002JA009491.

Shiokawa, K., Y. Otsuka, C. Ihara, T. Ogawa, and F. J. Rich (2003b), Ground and satellite observations of nighttime medium-scale traveling ionospheric disturbance at midlatitude, J. Geophys. Res., 108(A4), 1145, doi:10.1029/2002JA009639.

Shiokawa, K., Otsuka, Y., Ogawa, T., and Wilkinson, P.: Time evolution of high-altitude plasma bubbles imaged at geomagnetic conjugate points, Ann. Geophys., 22, 3137-3143, https://doi.org/10.5194/angeo-22-3137-2004, 2004. 
727

728

729

730

731

732

733

734

735

736

737

738

739

740

741

742

743

744

Shiokawa, K., Y. Otsuka, and T. Ogawa (2006), Quasiperiodic southward moving waves in 630-nm airglow images in the equatorial thermosphere, J. Geophys. Res., 111, A06301, doi:10.1029/2005JA011406.

Sun, L., J. Xu, W. Wang, W. Yuan, Q. Li, and C. Jiang (2016), A statistical analysis of equatorial plasma bubble structures based on an all-sky airglow imager network in China, J. Geophys. Res. Space Physics, 121, 11,495- 11,517, doi:10.1002/2016JA022950.

Suzuki, S., Shiokawa, K., Otsuka, Y., Ogawa, T., Nakamura, K., \& Nakamura, T. (2007). A concentric gravity wave structure in the mesospheric airglow images. Journal of Geophysical Research: Atmospheres, 112(D2).

Takeo, K. Shiokawa, H. Fujinami, Y. Otsuka, T. S. Matsuda, M. K. Ejiri, T. Nakamura, and M. Yamamoto (2017), Sixteen-year variation of horizontal phase velocity and propagation direction of mesospheric and thermospheric waves in airglow images at Shigaraki, Japan, J. Geophys. Res. Spce Physics, 122, 7849-9088,2017, doi:10.1002/2017JA023919

Tsai, L. C., Su, S. Y., Liu, C. H., Schuh, H., Wickert, J., \& Alizadeh, M. M. (2018). Global morphology of ionospheric sporadic E layer from the FormoSat3/COSMIC GPS radio occultation experiment. GPS Solutions, 22(4), 118. 
745 Tsunoda, R. T. (2006), On the coupling of layer instabilities in the nighttime midlatitude ionosphere, J. Geophys. Res., 111, A11304, doi:10.1029/2006JA011630.

747

748

Tsuchiya, S., Shiokawa, K., Fujinami, H., Otsuka, Y., Nakamura, T., Connors, M., ... \& Poddelsky, I. (2019). Three-Dimensional Fourier Analysis of the Phase Velocity Distributions of Mesospheric and Ionospheric Waves Based on Airglow Images Collected Over 10 Years: Comparison of Magadan, Russia, and Athabasca, Canada. Journal of Geophysical Research: Space Physics, 124(10), 8110-8124.

Tsugawa, T., N. Kotake, Y. Otsuka, and A. Saito (2007a), Medium-scale traveling ionospheric disturbances observed by GPS receiver network in Japan: A short review, GPS Solutions, 11, 139- 144, doi:10.1007/s10291-006-0045-5.

Tsugawa, T., Y. Otsuka, A. J. Coster, and A. Saito (2007b), Medium-scale traveling ionospheric disturbances detected with dense and wide TEC maps over North America, Geophys. Res. Lett., 34, L22101, doi:10.1029/2007GL031663.

Vadas, S. L., \& Fritts, D. C. (2009, January). Reconstruction of the gravity wave field from convective plumes via ray tracing. In Annales Geophysicae (Vol. 27, No. 1, pp. 147-177). Copernicus GmbH.

Vadas, S. L., and G. Crowley (2010), Sources of the traveling ionospheric disturbances observed by the ionospheric TIDDBIT sounder near Wallops Island on 30 October 2007, J. Geophys. Res., 115, A07324, doi:10.1029/2009JA015053. 
764 Whitehead, J. K. (1961), The formation of the sporadic-E layer in the temperate zones, J. Atmos. Terr. Phys., 20, 49-58, doi:10.1016/0021-9169(61)90097-6

766

767

768

769

770

771

772

773

774

775

776

777

778

779

780

781

Whitehead, J. D. (1989). Recent work on mid-latitude and equatorial sporadic-E. Journal of Atmospheric and Terrestrial Physics, 51(5), 401-424.

Yao, D., \& Makela, J. J. (2007). Analysis of equatorial plasma bubble zonal drift velocities in the pacific sector by imaging techniques. Annales Geophysicae, 25, 701-709.

Yeh, W. H., Liu, J. Y., Huang, C. Y., \& Chen, S. P. (2014). Explanation of the sporadicE layer formation by comparing FORMOSAT-3/COSMIC data with meteor and wind shear information. Journal of Geophysical Research: Atmospheres, 119(8), 4568-4579.

Yokoyama, T., D. L. Hysell, Y. Otsuka, and M. Yamamoto (2009), Three-dimensional simulation of the coupled Perkins and Es-layer instabilities in the nighttime midlatitude ionosphere, J. Geophys. Res., 114, A03308, doi:10.1029/2008JA013789.

Yokoyama, T., Shinagawa, H., \& Jin, H. (2014). Nonlinear growth, bifurcation, and pinching of equatorial plasma bubble simulated by three-dimensional highresolution bubble model. Journal of Geophysical Research: Space Physics, $119(12), 10-474$. 
783 Zhou, X., Holton, J. R., \& Mullendore, G. L. (2002). Forcing of secondary waves by breaking of gravity waves in the mesosphere. Journal of Geophysical Research: Atmospheres, 107(D7), ACL-3.

786

Zhou, Chen \& Tang, Qiong \& Song, Xiaoxiao \& Qing, Haiyin \& Liu, Yi \& Wang, Xiang \& Gu, Xudong \& Ni, Binbin \& Zhao, Zhengyu. (2017). A Statistical Analysis of Sporadic E layer Occurrence in the Mid-latitude China Region: Sporadic E layer Occurrence. Journal of Geophysical Research: Space Physics. 122. 10.1002/2016JA023135.

791

792

\section{$793 \quad$ Figure 1}

794 Identification of GPSTEC perturbations over Taiwan. (a) 60 min. high-pass filtered GNSS-TEC for

795 extracting the MSTID features. (b) Horizontal range of the ionospheric phenomena detection. The

796 latitude and longitude range are $20-30^{\circ} \mathrm{N}$ and $115-125^{\circ} \mathrm{E}$, respectively, with both zonal and meridional

797 resolution of $\sim 0.5$ degree.

798

$799 \quad$ Figure 2

800 Flowchart of the procedure of creating an SVM model for classifying different ionospheric conditions. 


\section{Figure 3}

803 Local time and seasonal variations of the MSTID obtained from 3D-FFT and SVM analysis over Taiwan

804 during 2013-2015. It is divided into 10 days and half an hour per grid for calculating the occurrence rate

805 of southward MSTIDs in (a) 2013 (b) 2014 (c) 2015 year and northward MSTIDs in (d) 2013 (e) 2014

806 (f) 2015 year. The propagation direction of MSTIDs is compared to the dusk terminator (black line) in

807

(g) 2013 (h) 2014 (i) 2015 year.

808

$809 \quad$ Figure 4

810 Daytime and nighttime MSTID propagation over Japan and Taiwan. (a) An example of typical daytime

811 MSTIDs over mid- and low-latitude region, and (b) example showing nighttime MSTIDs propagating

812 from Japan to Taiwan. (c) The filtered TEC perturbations, along the orange dashed line in Figure 4b,

813 organized as time-distance map.

814

$815 \quad$ Figure 5

816 A comparison between propagation direction and normalized PSD of MSTIDs with a dusk terminator

817 (black line) over Taiwan. The southwestward MSTIDs (deep blue color) in the years (a) 2013 (b) 2014

818 (c) 2015 is in good agreement with the PSD with greater value (red color) in (d) 2013 (e) 2014 (f) 2015 ,

819 suggesting that the southwestward MSTIDs have greater amplitudes than those propagating in other

820 directions. 


\section{$822 \quad$ Figure 6}

823 Comparison of EsL and MSTID occurrence. (a) The occurrence rate of EsL detected by FORMOSAT-

824 3/COSMIC RO over Taiwan and the conjugate region of Taiwan in nighttime during 2013-2015. (b) The

825 propagation direction of MSTIDs over Taiwan in nighttime during 2013-2015. (c) The occurrence rate

826 of southward MSTIDs over Taiwan in nighttime during 2013-2015. (d) The occurrence rate of northward

827 MSTIDs over Taiwan in nighttime during 2013-2015. The black lines indicate the dusk terminator.

828

$829 \quad$ Figure 7

830 The occurrence rate of (a) northwestward, (b) northward and (c) northeastward MSTIDs over Taiwan in

831 nighttime during 2013-2015. The black lines indicate the dusk terminator.

832

$833 \quad$ Figure 8

834 The $\lambda 557 \mathrm{~nm}$ airglow images derived from Tainan Astronomical Education Area on 29 April 2020.

835

836 Figure 9

837 Two-dimensional filtered GNSS-TEC maps over Taiwan on 29 April 2020.

838

$839 \quad$ Figure 10

840 Examples of (a) Zonal Wind and (b) Meridional Wind derived from high-resolution WACCM at 5.8e-4

841 hpa at 14:00 UT in 9 July. The yellow arrows indicate the propagation direction of the visible AGWs. (c)

842 Zonal wind and (d) Meridional Wind-time-distance map along the yellow arrow in Figure 10a and 10b, 
843 respectively. Each pair of the red symbols indicates an AGWs event. The numbers above the symbols

844 represent the phase velocity of the AGWs.

845

\section{$846 \quad$ Figure 11}

847 Comparison between the propagation directions of MSTIDs and the AGWs derived from WACCM. The

848 gray lines are the MSTID propagation direction during 2013-2015, and the blue line represents the

849 averaged value during 2013-2015. The red line with stars displays the corresponding WACCM results.

850 
Figures

(a)

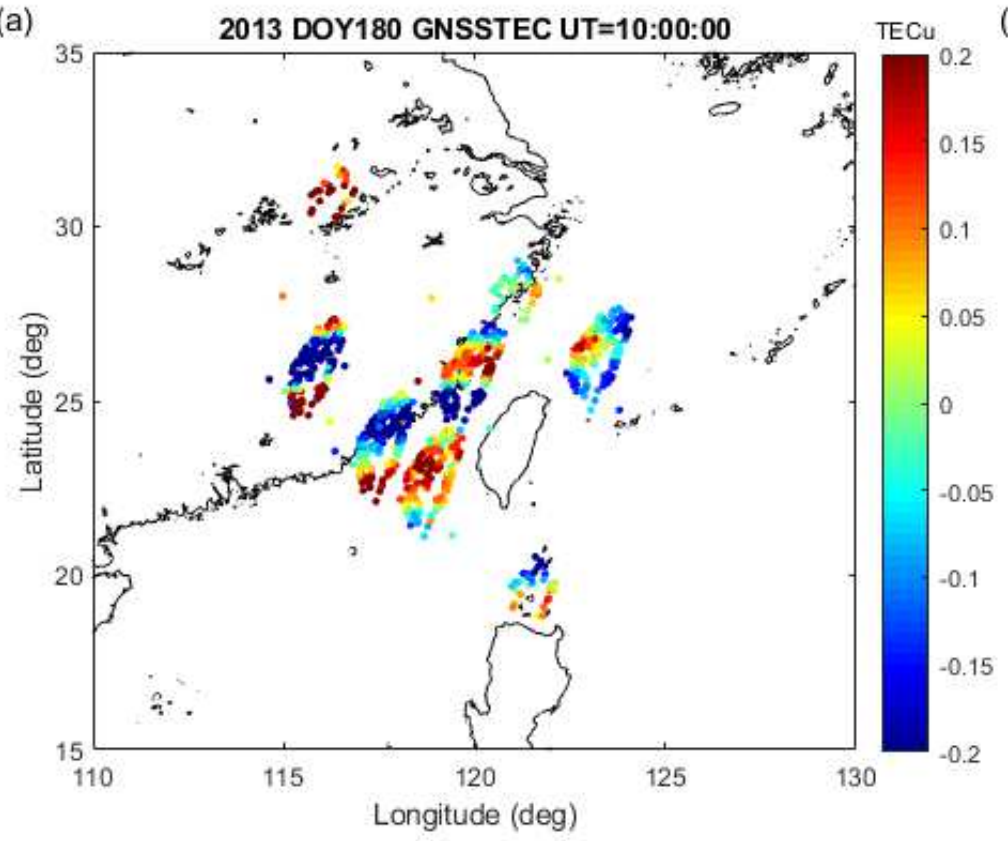

(b)

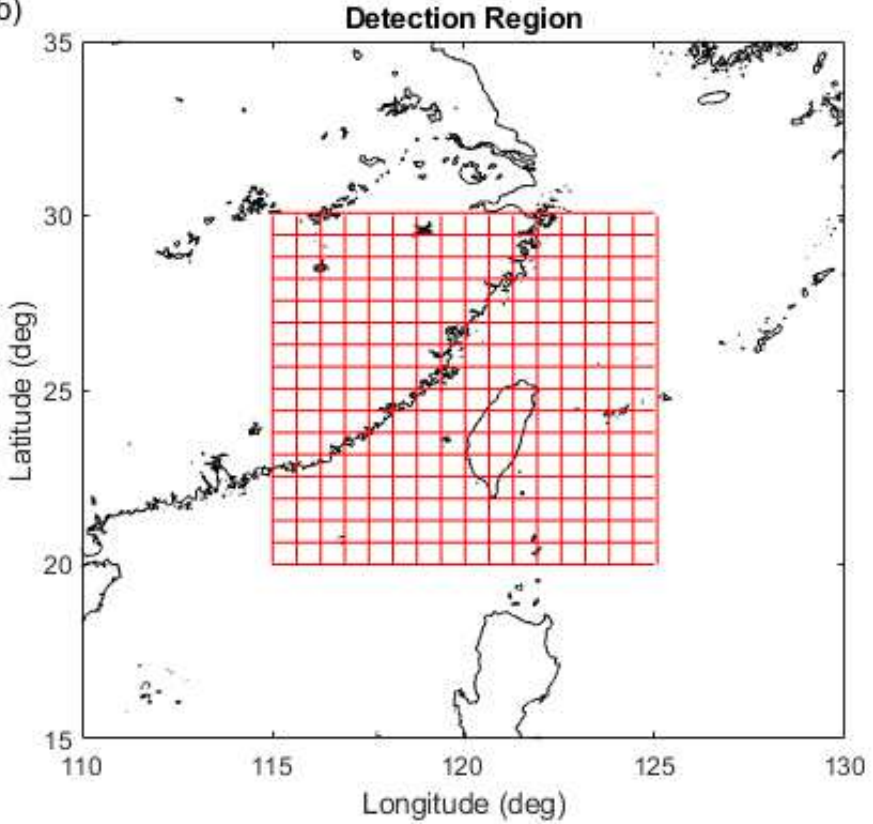

Figure 1

Identification of GPSTEC perturbations over Taiwan. (a) 60 min. high-pass filtered GNSS-TEC for extracting the MSTID features. (b) Horizontal range of the ionospheric phenomena detection. The latitude and longitude range are $20-30^{\circ} \mathrm{N}$ and $115-125^{\circ} \mathrm{E}$, respectively, with both zonal and meridional resolution of $\sim 0.5$ degree. 


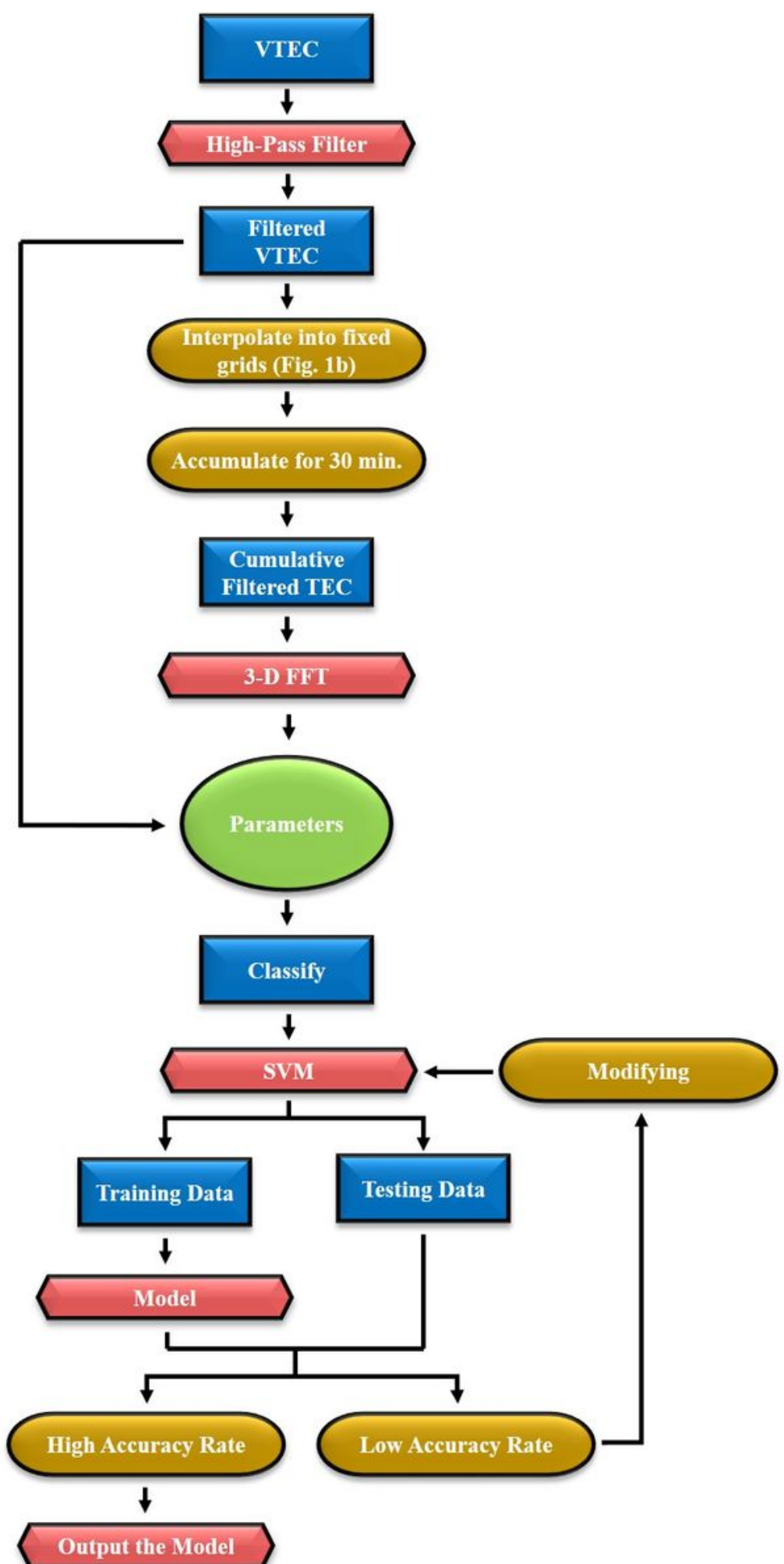

\section{Figure 2}

Flowchart of the procedure of creating an SVM model for classifying different ionospheric conditions. 


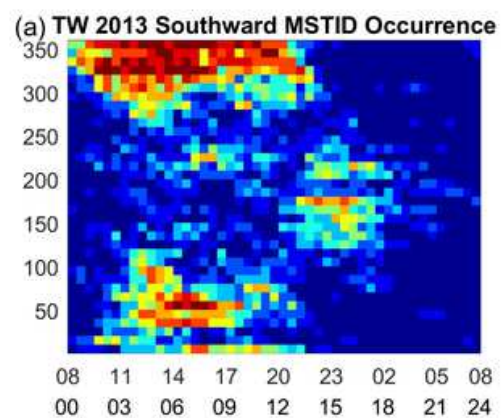

(d) TW 2013 Northward MSTID Occurrence
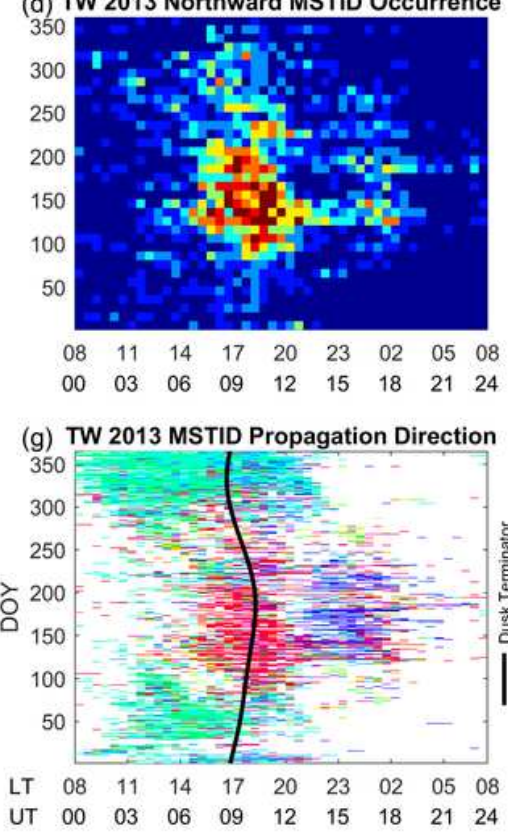

(b) TW 2014 Southward MSTID Occurrence

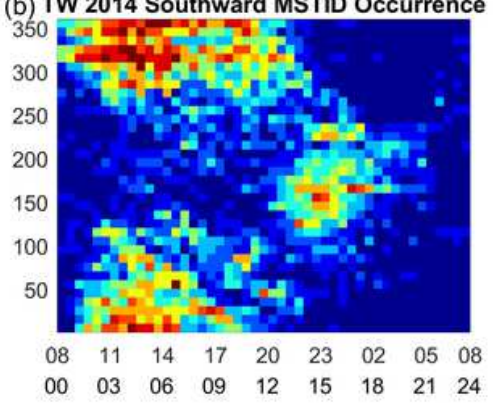

(e) TW 2014 Northward MSTID Occurrence

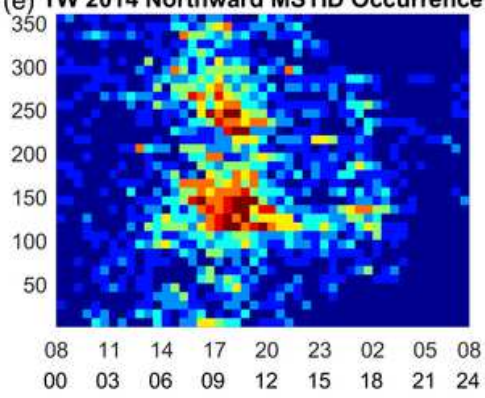

(h) TW 2014 MSTID Propagation Direction

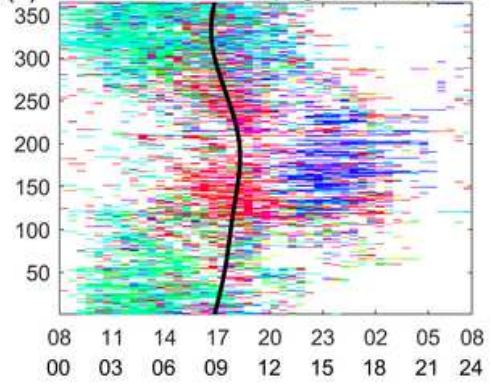

(c) TW 2015 Southward MSTID Occurrence

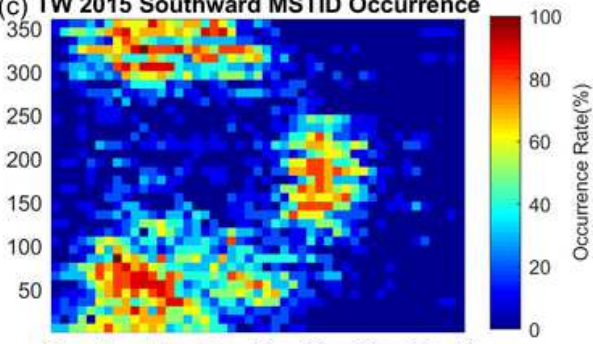

$\begin{array}{lllllllll}08 & 11 & 14 & 17 & 20 & 23 & 02 & 05 & 08\end{array}$

$\begin{array}{lllllllll}00 & 03 & 06 & 09 & 12 & 15 & 18 & 21 & 24\end{array}$
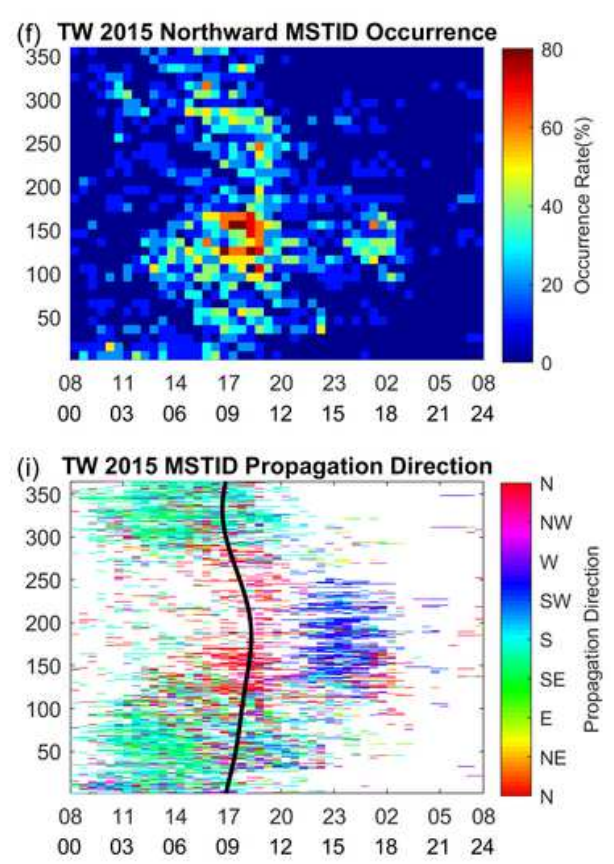

\section{Figure 3}

Local time and seasonal variations of the MSTID obtained from 3D-FFT and SVM analysis over Taiwan during 2013-2015. It is divided into 10 days and half an hour per grid for calculating the occurrence rate of southward MSTIDs in (a) 2013 (b) 2014 (c) 2015 year and northward MSTIDs in (d) 2013 (e) 2014 (f) 2015 year. The propagation direction of MSTIDs is compared to the dusk terminator (black line) in (g) 2013 (h) 2014 (i) 2015 year. 
(a)

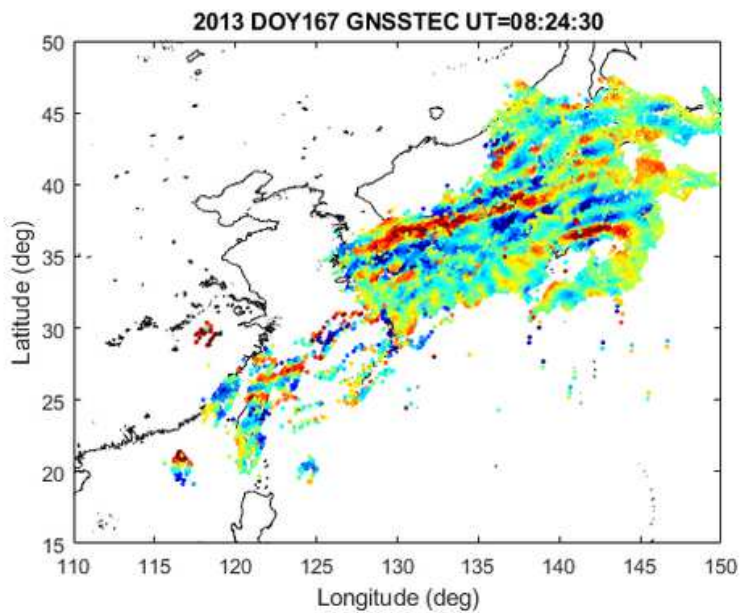

(b)

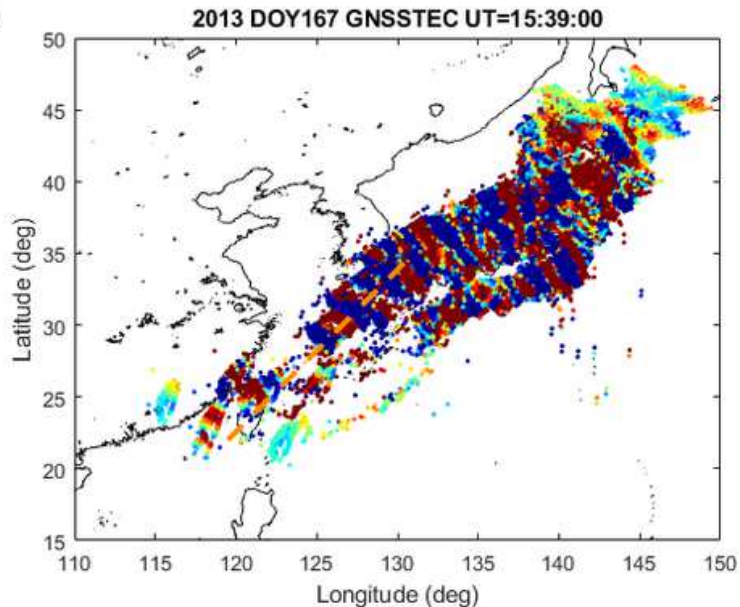

(c)

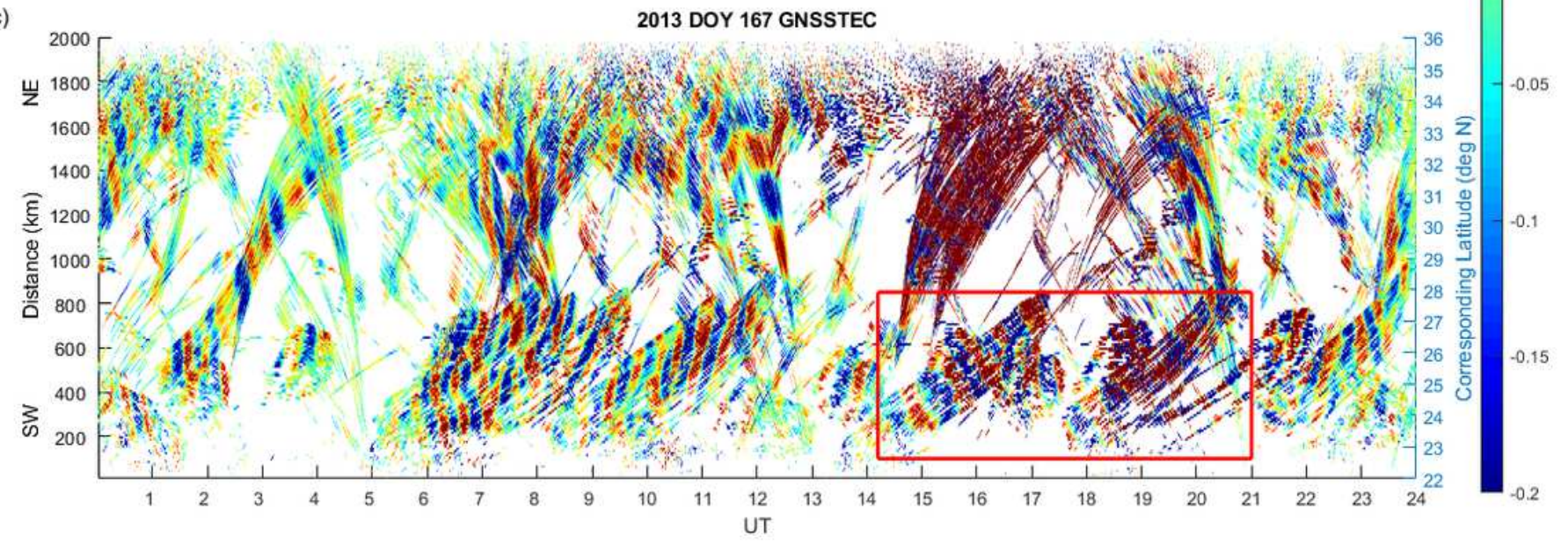

Figure 4

Daytime and nighttime MSTID propagation over Japan and Taiwan. (a) An example of typical daytime MSTIDs over mid- and low-latitude region, and (b) example showing nighttime MSTIDs propagating from Japan to Taiwan. (c) The filtered TEC perturbations, along the orange dashed line in Figure 4b, organized as time-distance map. 

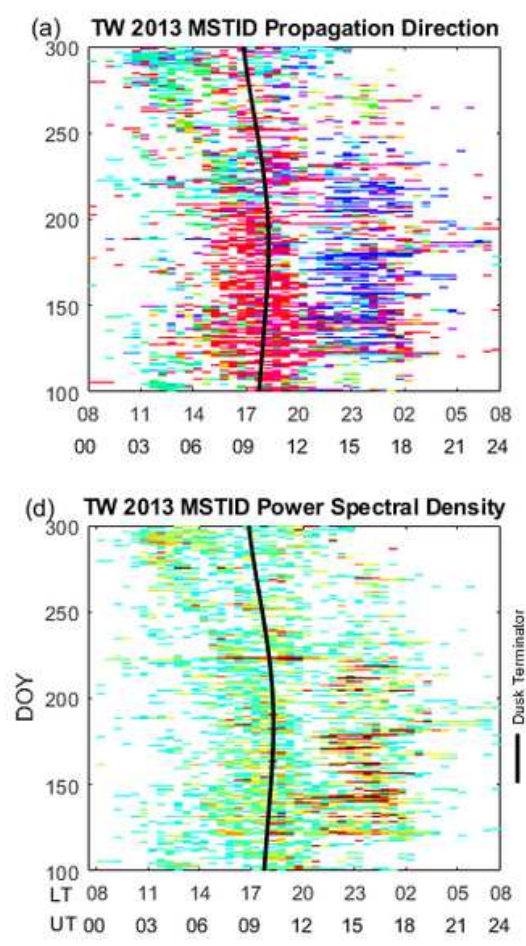

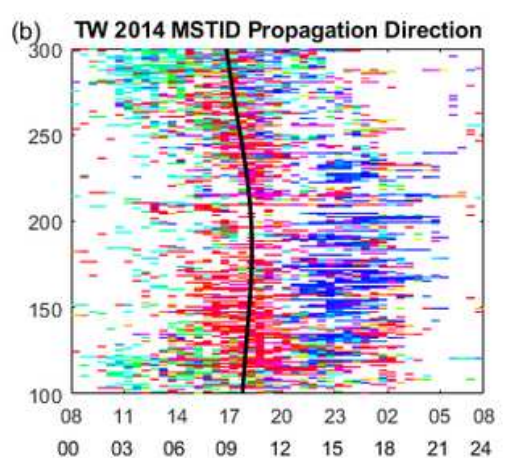

(e) TW 2014 MSTID Power Spectral Density

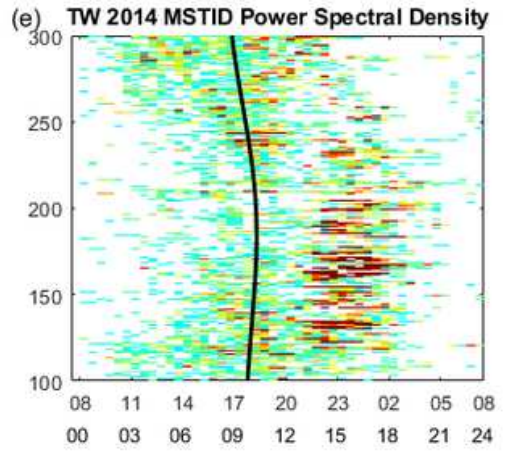

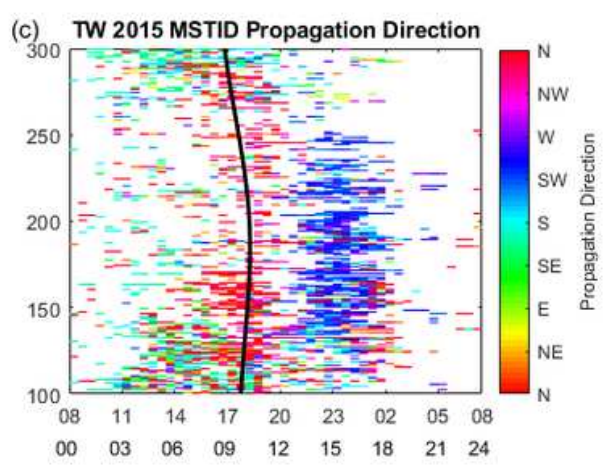

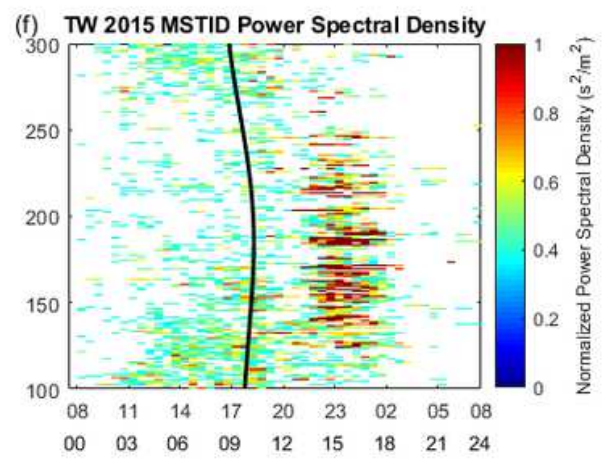

\section{Figure 5}

A comparison between propagation direction and normalized PSD of MSTIDs with a dusk terminator (black line) over Taiwan. The southwestward MSTIDs (deep blue color) in the years (a) 2013 (b) 2014 (c) 2015 is in good agreement with the PSD with greater value (red color) in (d) 2013 (e) 2014 (f) 2015, suggesting that the southwestward MSTIDs have greater amplitudes than those propagating in other directions. 
(a) TW \& Conjugate Region 2013-2015 EsL Activity

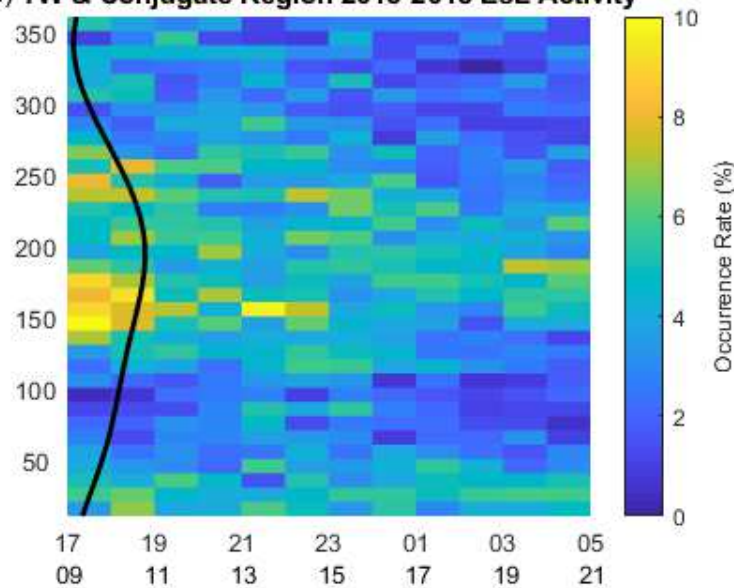

(c) TW 2013-2015 Southward MSTID Activity

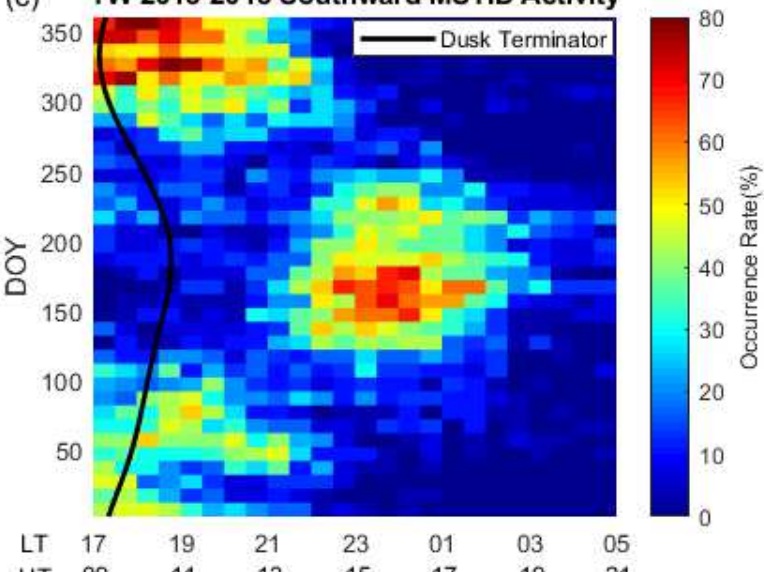

(b) TW 2013-2015 MSTID Propagation Direction

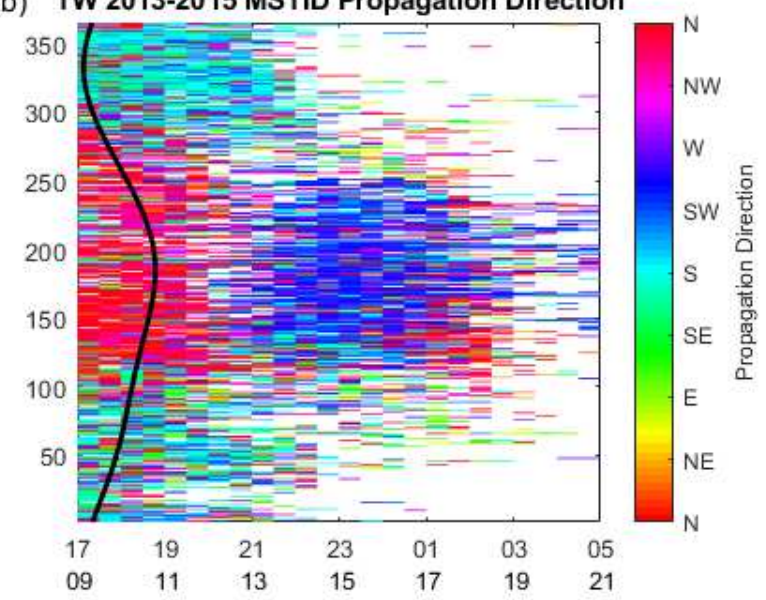

(d) TW 2013-2015 Northward MSTID Activity

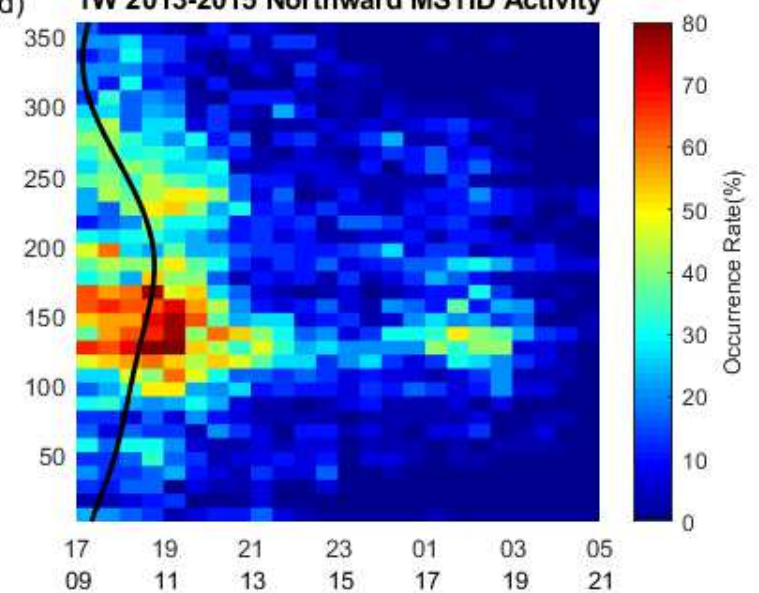

Figure 6

Comparison of EsL and MSTID occurrence. (a) The occurrence rate of EsL detected by FORMOSAT3/COSMIC RO over Taiwan and the conjugate region of Taiwan in nighttime during 2013-2015. (b) The propagation direction of MSTIDs over Taiwan in nighttime during 2013-2015. (c) The occurrence rate of southward MSTIDs over Taiwan in nighttime during 2013-2015. (d) The occurrence rate of northward MSTIDs over Taiwan in nighttime during 2013-2015. The black lines indicate the dusk terminator.
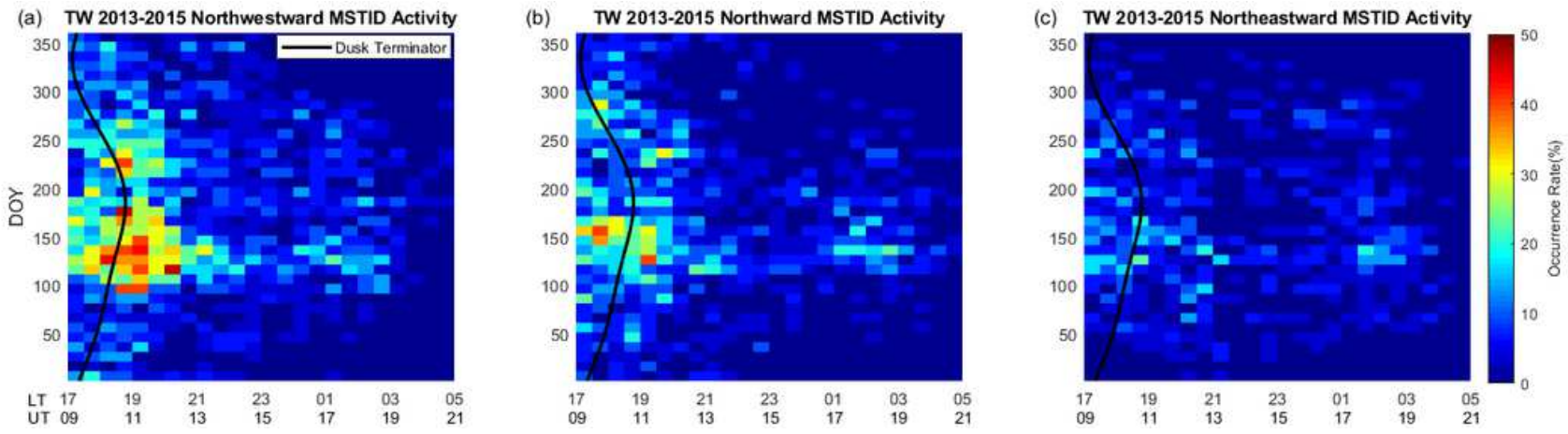
The occurrence rate of (a) northwestward, (b) northward and (c) northeastward MSTIDs over Taiwan in nighttime during 2013-2015. The black lines indicate the dusk terminator.

(a)

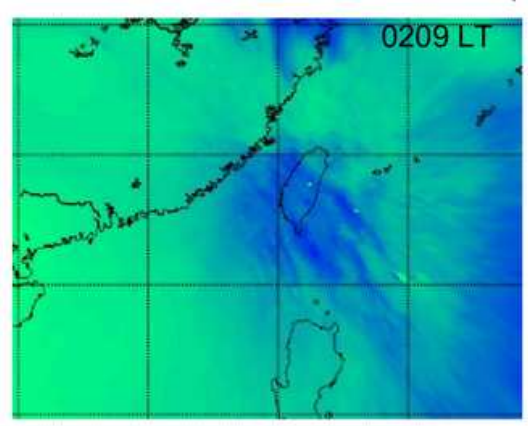

(b)

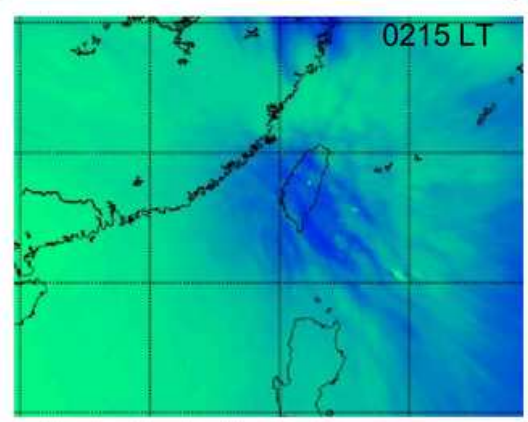

(c)

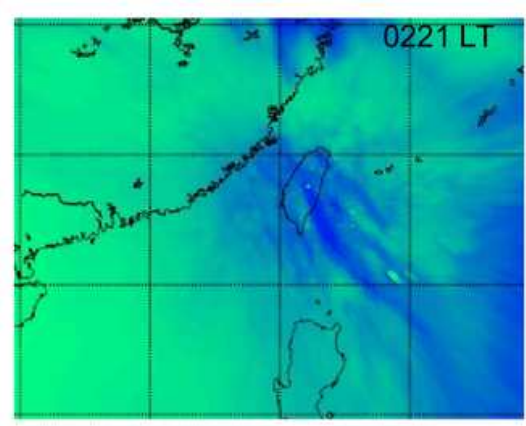

(f)

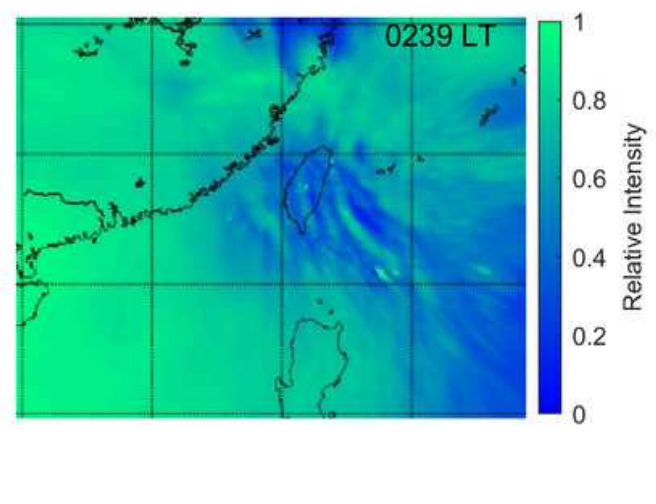

\section{Figure 8}

(e)
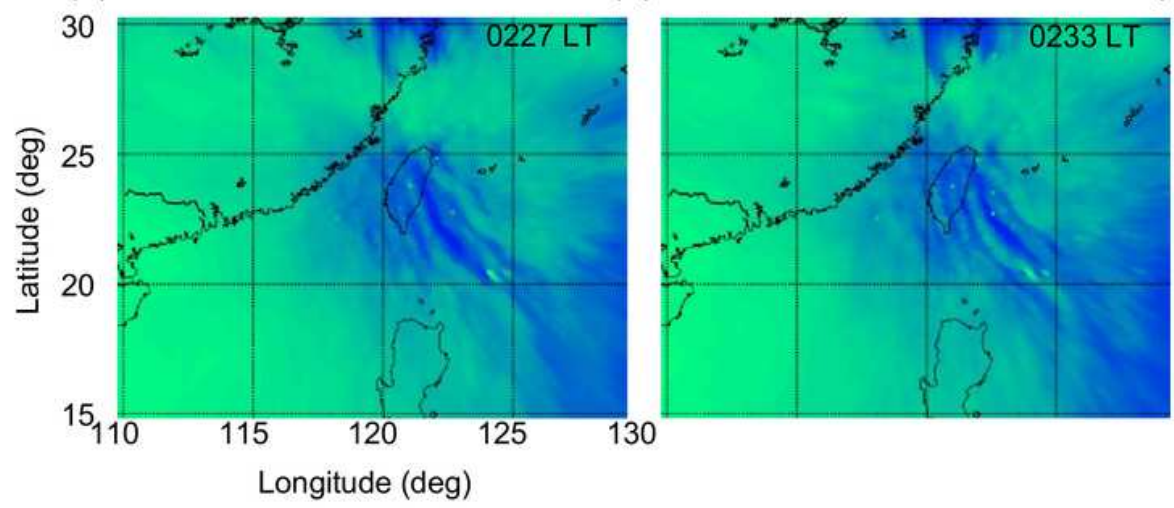

The $\lambda 557 \mathrm{~nm}$ airglow images derived from Tainan Astronomical Education Area on 29 April 2020.
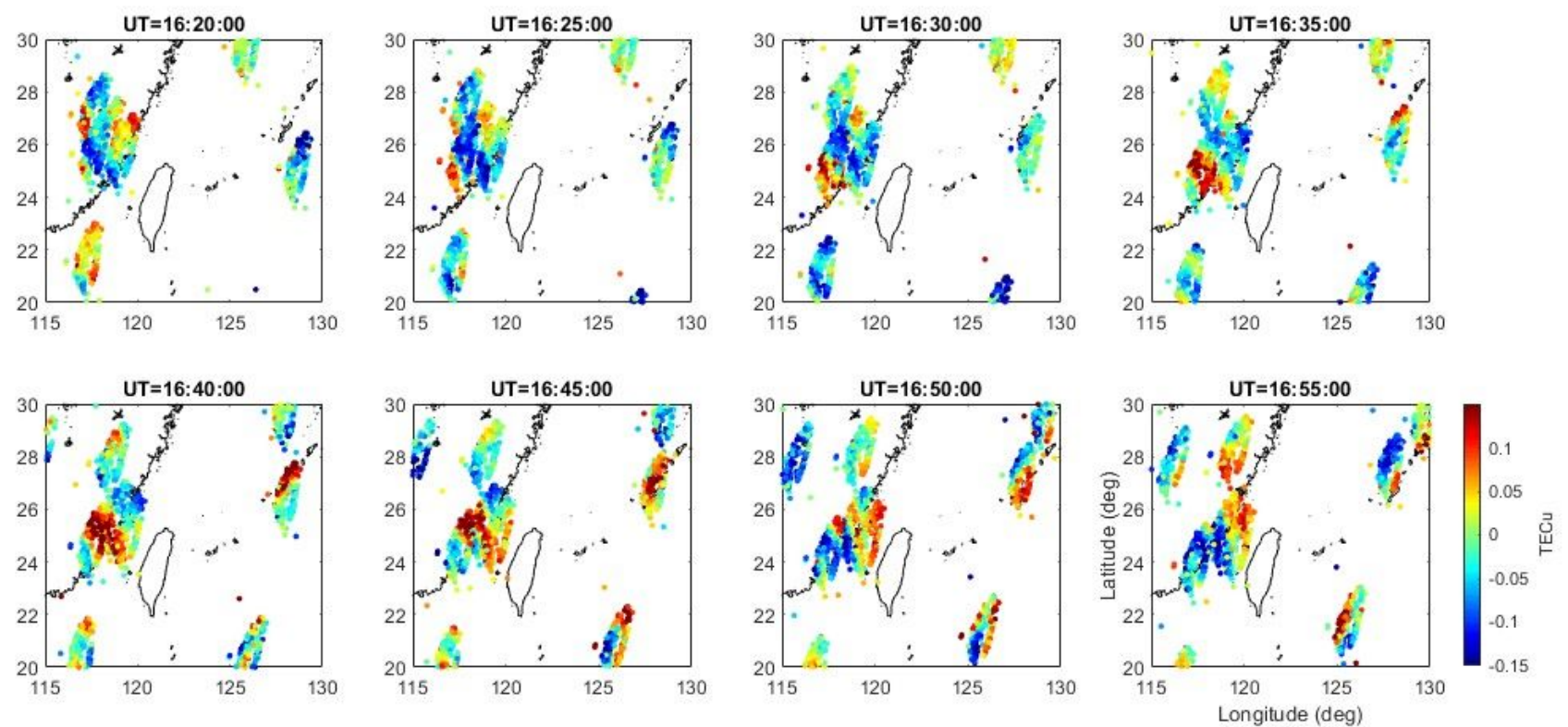


\section{Figure 9}

Two-dimensional filtered GNSS-TEC maps over Taiwan on 29 April 2020.

(a) ${ }_{45}$ WACCM 9 July UT : 14 Zonal Wind at $5.8 \mathrm{e}-4 \mathrm{hpa}$

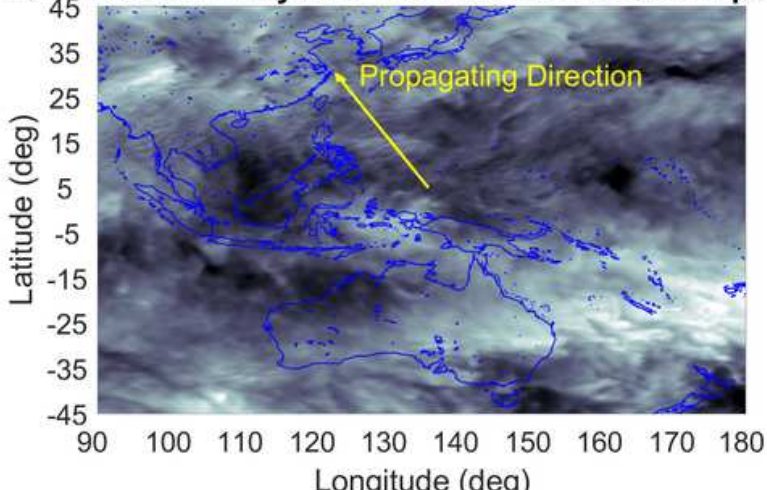

(b) WACCM 9 July UT : 14 Meridional Wind at 5.8e-4hpa

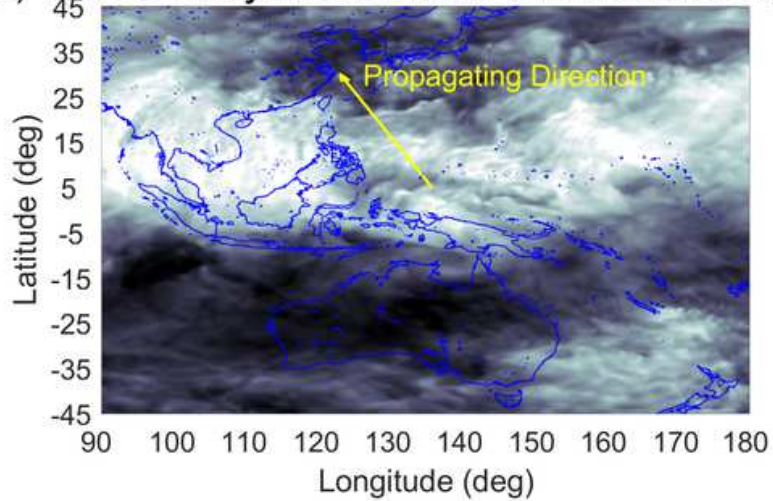

(c)

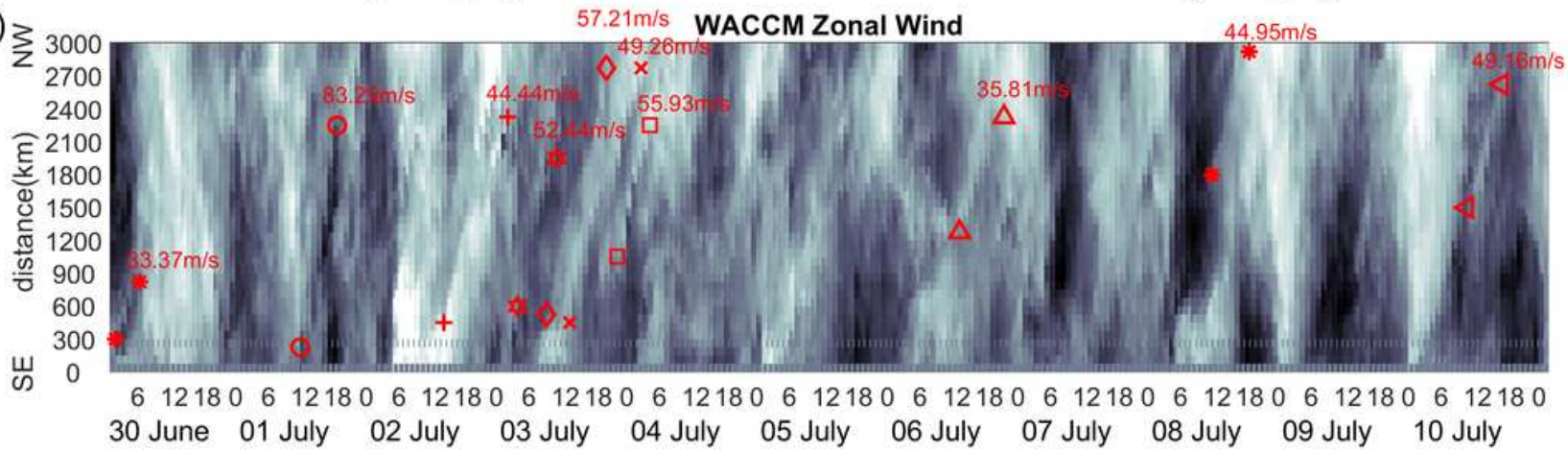

(d) 30 June 01 July 02 July $\quad 03$ July 04 July $\quad 05$ July 06 July $\quad 07$ July 08 July 09 July 10 July

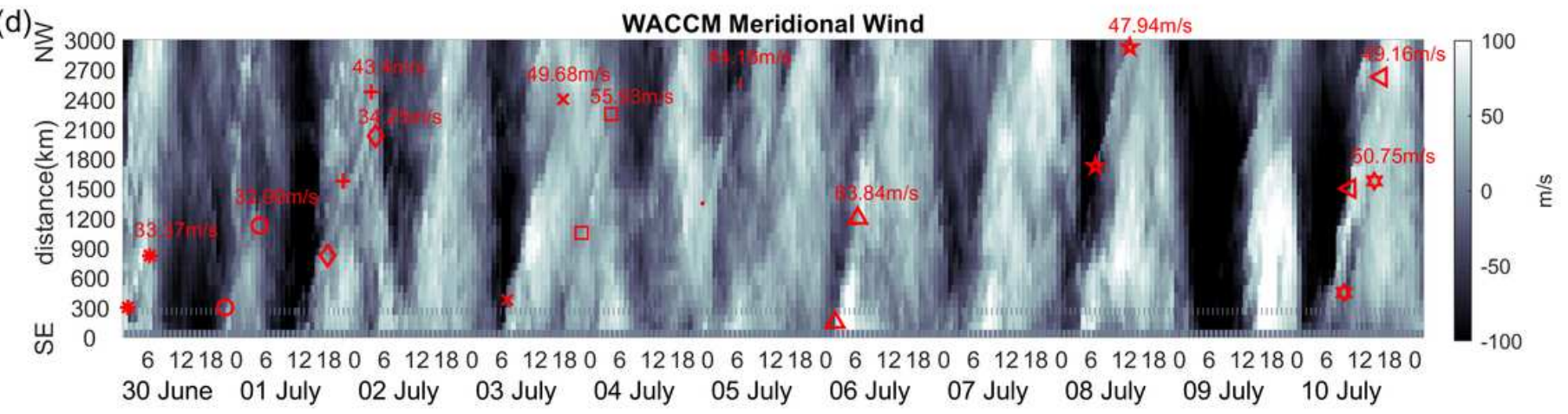

\section{Figure 10}

Examples of (a) Zonal Wind and (b) Meridional Wind derived from high-resolution WACCM at 5.8e-4 hpa at 14:00 UT in 9 July. The yellow arrows indicate the propagation direction of the visible AGWs. (c) Zonal wind and (d) Meridional Wind-time-distance map along the yellow arrow in Figure 10a and 10b, respectively. Each pair of the red symbols indicates an AGWs event. The numbers above the symbols represent the phase velocity of the AGWs. 


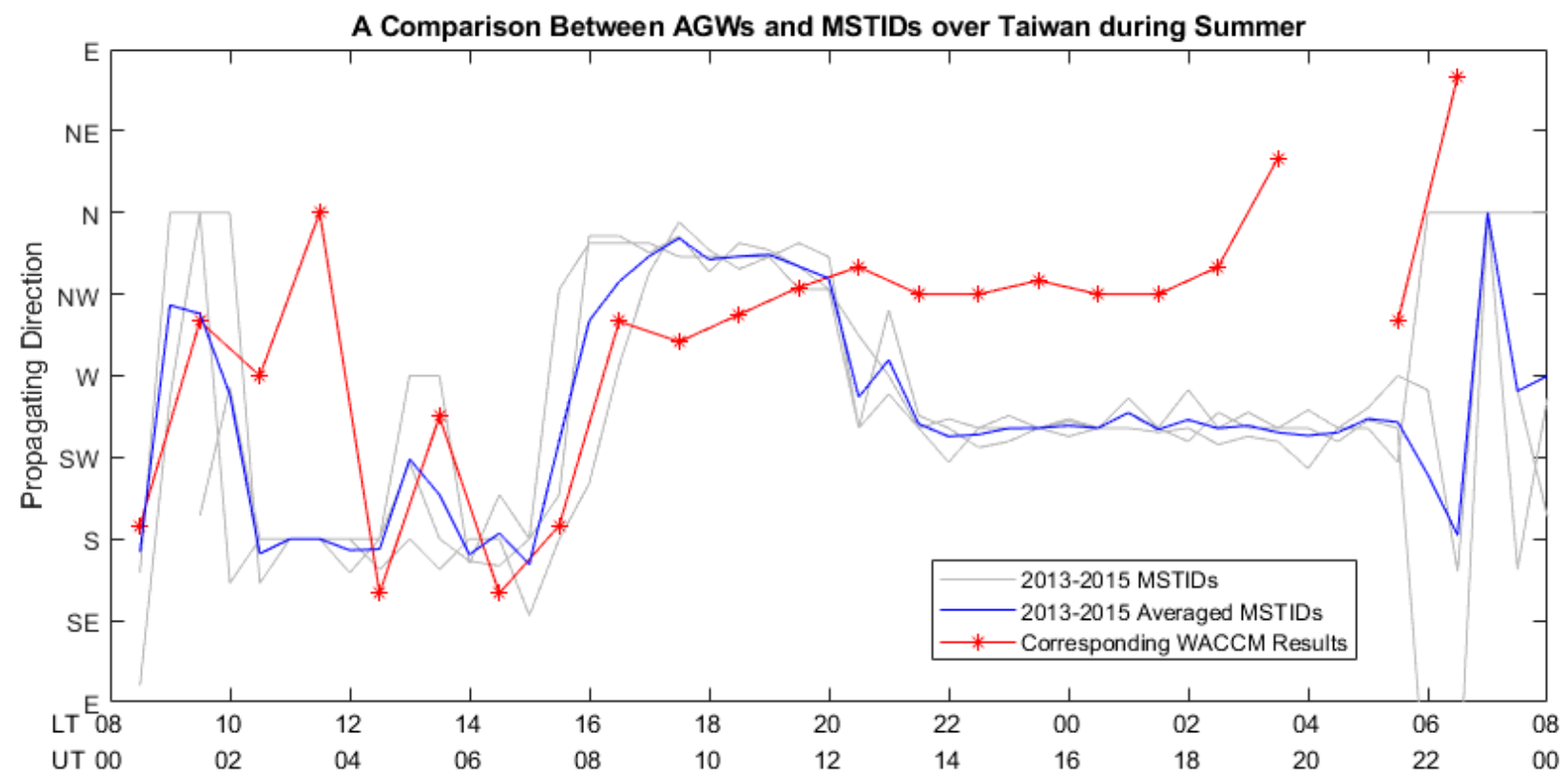

\section{Figure 11}

Comparison between the propagation directions of MSTIDs and the AGWs derived from WACCM. The gray lines are the MSTID propagation direction during 2013-2015, and the blue line represents the averaged value during 2013-2015. The red line with stars displays the corresponding WACCM results.

\section{Supplementary Files}

This is a list of supplementary files associated with this preprint. Click to download.

- grapjicalabstract.png 